\title{
GTAG- and CGTC-tagged palindromic DNA repeats in prokaryotes
}

\author{
Pier Paolo Di Nocera*, Eliana De Gregorio and Francesco Rocco
}

\begin{abstract}
Background: REPs (Repetitive Extragenic Palindromes) are small (20-40 bp) palindromic repeats found in high copies in some prokaryotic genomes, hypothesized to play a role in DNA supercoiling, transcription termination, mRNA stabilization.

Results: We have monitored a large number of REP elements in prokaryotic genomes, and found that most can be sorted into two large DNA super-families, as they feature at one end unpaired motifs fitting either the GTAG or the CGTC consensus. Tagged REPs have been identified in $>80$ species in 8 different phyla. GTAG and CGTC repeats reside predominantly in microorganisms of the gamma and alpha division of Proteobacteria, respectively. However, the identification of members of both super- families in deeper branching phyla such Cyanobacteria and Planctomycetes supports the notion that REPs are old components of the bacterial chromosome. On the basis of sequence content and overall structure, GTAG and CGTC repeats have been assigned to 24 and 4 families, respectively. Of these, some are species-specific, others reside in multiple species, and several organisms contain different REP types. In many families, most units are close to each other in opposite orientation, and may potentially fold into larger secondary structures. In different REP-rich genomes the repeats are predominantly located between unidirectionally and convergently transcribed ORFs. REPs are predominantly located downstream from coding regions, and many are plausibly transcribed and function as RNA elements. REPs located inside genes have been identified in several species. Many lie within replication and global genome repair genes. It has been hypothesized that GTAG REPs are miniature transposons mobilized by specific transposases known as RAYTs (REP associated tyrosine transposases). RAYT genes are flanked either by GTAG repeats or by long terminal inverted repeats (TIRs) unrelated to GTAG repeats. Moderately abundant families of TIRs have been identified in multiple species.
\end{abstract}

Conclusions: CGTC REPs apparently lack a dedicated transposase. Future work will clarify whether these elements may be mobilized by RAYTs or other transposases, and assess if de-novo formation of either GTAG or CGTC repeats type still occurs.

Keywords: Palindromic sequences, Repeated DNA families, RNA hairpins, Transposases, Mobile DNA, Intragenic DNA elements

\section{Background}

Repetitive sequences occur in large quantities in eukaryotic cells, but they also constitute a significant fraction of the DNA of many prokaryotic genomes. According to the sizes, prokaryotic DNA repeats may be broadly sorted into two main groups. Large repeats are mostly represented by IS (Insertion Sequences). IS measure $0.8-2 \mathrm{~kb}$, feature terminal inverted repeats (TIRs)

\footnotetext{
* Correspondence: dinocera@unina.it

Dipartimento di Medicina Molecolare e Biotecnologie Mediche, Università Federico II, Napoli, Via S. Pansini 5 80131, Naples, Italy
}

and encode endonucleases which interact with TIRs promoting IS mobilization [1,2]. Small repeats vary in size from 20 to $300 \mathrm{bp}$, have different structures and can be sorted into a few distinct classes [3]. One is represented by tandemly arranged repeats called CRISPRs (Clustered Regularly Interspaced Short Palindromic Repeats). CRISPRs measure 24 to $48 \mathrm{bp}$, and are located at one or more loci in several prokaryotic genomes, separated by regularly sized, non-repetitive sequences, which originate from the processing of plasmid and/or bacteriophage DNA, mediated by CRISPR-associated proteins. Spacer sequences
C Biomed Central 
serve as a 'memory' of past exposures to foreign DNA, and are used to recognize and silence exogenous genetic elements in a manner analogous to RNAi in eukaryotic organisms [4]. CRISPRs usually show some dyad symmetry but are not truly palindromic, and thus structurally differ from the elements called REPs (Repetitive Extragenic Palindromes). REPs are 20-40 bp long palindromic repeats, early described as an abundant component of the Escherichia coli genome (reviewed in [5]), and later shown to represent a significant fraction of the extragenic space of many prokaryotic genomes [6-9]. REPs are found as single units, but also close to each other, and pairs as larger clusters of REPs are referred to as BIME (Bacterial Interspersed Mosaic Elements). REPs and BIMEs have been hypothesized to play a role in processes as diverse as DNA supercoiling, transcription termination, mRNA stabilization [10,11]. Moreover, REPs can affect genome plasticity, by functioning as targets for insertion of IS sequences in Pseudomonas, Neisseria and Sinorhizobium Genus [12]. REP-like elements known as RPEs (Repetitive Palindromic Elements) were identified in the genome of the obligate intracellular bacterium $R$. conorii, and many found surprisingly inserted in-frame within open reading frames which likely encode functional proteins $[13,14]$. The third group of small prokaryotic DNA repeats is constituted by MITEs (Miniature Inverted-repeat Transposable Elements), 70-300 bp elements which resemble degenerated ISs, as they feature 15-30 bp TIRs, but have no coding capacity. The group of bacterial MITEs includes RUP elements in Streptococcus pneumoniae [15], NEMIS elements in Neisseria meningitidis [16,17], Bcr1 elements in Bacillus cereus [18], ERIC and YPAl elements in Yersinia enterocolitica [19,20], Nezha elements in Cyanobacteria [21], EFAR elements in Enterococci [22]. MITEs are often inserted next to coding sequences, are transcribed and influence the expression of neighboring genes by folding into robust secondary structures, which can either stabilize the mRNA, or alternatively accelerate its degradation [23]. MITEs can be mobilized by transposases recognizing their TIRs [15,16,24]. REPs may be miniature non-autonomous mobile DNA elements as well, since they are often associated to genes encoding transposases of the IS200/IS605 family, accordingly called RAYTs (REP-associated tyrosine transposases; ref. [25]).

REPs characteristically terminate at one end with the tetranucleotide GTAG $[9,25,26]$. Intriguingly, we found that $R$. conorii RPE sequences terminate at one end with the tetranucleotide CGTC. We have identified in prokaryotic genomes several families of short palindromic repeats alternatively tagged at one end either by GTAG or CGTC tetranucleotides. Multiple families of either or both repeat types reside in some microorganisms. Structure, genomic organization, chromosomal arrangement, degree of inter- and intraspecies variation, pattern of interspersion with coding regions of all these sequences are reported. The role played by specific transposases in the formation and maintenance of the various repeats is discussed. In several species, RAYT genes are not flanked by REPs, but rather by long TIRs. In some of them, moderately abundant families of TIR repeats have been identified.

\section{Results}

Short SLSs tagged at one end by the tetranucleotide GTAG or CGTC mark the genome of several microorganisms. According to their branching patterns in the $16 \mathrm{~S}$ rRNA trees, bacteria are divided into main phyla. GTAG repeats have been identified in microorganisms belonging to the Proteobacteria, Cyanobacteria, and Chloroflexi phyla, and the PVC (Planctomycetes, Verrucomicrobia and Chlamydiales; see ref. [27]) superphylum. GTAG repeats were found in all divisions (alpha to epsilon) of Proteobacteria, but predominate in bacteria of the late-branching [28] gamma division. Cyanobacteria occur as unicellular and multicellular microorganisms [29], and GTAG elements were found in both cell types. CGTC repeats were identified in microorganisms belonging to 5 phyla: Proteobacteria, Chlorobi, Bacteroidetes, Spirochaetes, Thermotogae. In contrast to GTAG repeats, CGTC repeats predominate in Proteobacteria of the alpha division. Most reside in free-living organisms, but some have been identified in obligate intracellular bacteria, such Wolbachia and Rickettsiae. CGTC and GTAG repeats coexist in Neisseriae, Bradyrhizobium, Rhodopseudomonas palustris, Sulfurovum sp. NB37-1, and Coxiella burnetii. This bacterium substantially differs from typical obligate intracellular bacteria because having a relatively large genome and most metabolic pathways intact, and may indeed be considered a facultative intracellular bacterium [30].

Features and properties of the identified GTAG and CGTC repeat families are described below.

\section{GTAG families}

GTAG families have been sorted into 24 families (Figure 1). The classification takes into account changes of the stems, in terms of length (6-13 bp) and base composition, as changes of the loops, which measure 23 bp in many families, but vary in length among members of some families (Figure 1). Some GTAG families are restricted to one species only, others reside in multiple species of the same genus or order, as in evolutionary distant microorganisms. Repeats conserved in a genus have been analyzed in detail in strains of one or more species selected in the past for similar studies by other investigators. REPs identified in Escherichia [5] and Pseudomonas [6,7] genomes correspond to some of the GTAG-3 and GTAG-1 families listed in Figure 1, 


\section{GTAG families}

\begin{tabular}{|c|c|c|c|c|c|c|c|c|c|c|c|c|c|c|c|}
\hline & & & & & & & & & & & $\mathbf{S}$ & & $\mathbf{D}$ & & $\mathbf{G}$ \\
\hline Phylum & & Order & Species & & & Stem & & - Loop & & Stem & & HH & TT & HT & \\
\hline & & & Pseudomonas putida & & GTRG GA & $\overline{\text { GCGGGY }}$ & & KY & & RCCCGC GAA & 178 & 88 & 234 & 1 & 250 \\
\hline & & & Pseudomonas entomophila & & GTAG GA & GCSGVY & & TY & & RBCSGC GAW & 185 & 283 & 285 & 3 & 43 \\
\hline & & Dseoudomonodales $5 \mathrm{C}$ & Pseudomonas mendocina & & GTRG GA & GSGGMT & & TY & & AKCCSC GAN & 114 & 34 & 19 & 6 & 20 \\
\hline & & Pseudomonadales & Pseudomonas fluorescens & & GYAG GA & GCBRGC & & TT & & GCYVGC GAA & 207 & 162 & 76 & 3 & 280 \\
\hline & & & Pseudomonas syringae & & GTRG GA & GYGRRC & & TT & & GYYCRC GAA & 53 & 24 & 3 & 2 & 285 \\
\hline & $\gamma$ & & Azotobacter vinelandii & 1 & GYRG GA & GCGGAT & & $\mathrm{TC}$ & & ATCCGC GAY & 29 & 5 & 1 & - & - \\
\hline & & Chromatiales & Thioalkalivibrio K90mix & & GTRG GA & GCSKGC & & TY & & GCMSGC GAA & 17 & 12 & 1 & 1 & - \\
\hline & & Xanthomonadales & Xanthomonas oryzae & & GTAG GA & GCGSSC & & YY & & GSSCGC GAY & 15 & 14 & - & - & 6 \\
\hline & & Aantinomonadáles & Xanthomonas campestris & & GTAG GA & GCGSSC & & YY & & GSSCGC GAN & 35 & 24 & 3 & 3 & 210 \\
\hline & & Alteromonadales & Shewanella sediminis & & GTAG GA & SCGGCT & & TT & & AGCCGS GAA & 81 & 1 & 1 & 12 & 28 \\
\hline & $\beta$ & Rhodocyclales & Thauera MZ1T & & GTRG GA & GCGAC & & GCVA & & GTCGC GAY & 60 & 17 & 28 & 7 & 499 \\
\hline & $\gamma$ & Alteromonadales & Shewanella halifaxensis & 2 & GTAG GT & YGGSMT & & TT & & AKSCCR TCA & 30 & 8 & - & - & 12 \\
\hline & $\gamma$ & Oceanospirillales & Marinomonas MWYL1 & 2 & GTAG GT & CGGCCT & & TY & & AGGCCG TCA & 11 & - & - & 3 & 28 \\
\hline & $\gamma$ & Pseudomonadales & Pseudomonas mendocina & & GTAG & CCCGGAT & & $\mathrm{GCA}$ & & ATCCGGG & 59 & 37 & 4 & - & 3 \\
\hline & $\gamma$ & Legionellales & Coxiella burnetii & & GTAG & CCCGTAT & $G(V) A$ & & GRA & ATACGGG & 75 & 66 & 1 & - & - \\
\hline & $\alpha$ & Rhizobiales & Rhodopseudomonas palustris & & GTAG & CCCGSAT & $G(V) A$ & $\mathrm{GC}$ GnA GC & GMM & ATSCGGG & 16 & 25 & 1 & 1 & 61 \\
\hline & $\alpha$ & Rhizobiales & Bradyrhizobium ORS278 & & GTAG & CCCGSAT & $\mathrm{GA}$ & & GAY & ATSCGGG & 24 & 67 & - & - & - \\
\hline & & & [Cronobacter sakazakii & & GTAG & GGYGGGT & $\mathrm{AA}$ & GC $\mathrm{n} 2-4 \quad \mathrm{GC}$ & GC & ACCCRCC & 22 & - & 27 & 11 & 323 \\
\hline & & & Enterobacter cloacae & 3 & GTAG & GCCSGRT & AA & & $\mathrm{GCC}$ & AYCSGGC & 92 & 2 & 35 & - & - \\
\hline Proteobacteria & & & Citrobacter koseri & & GTAG & GCCBGRT & $A A$ & GC n2-4 GC & GCC & AYCVGGC & 207 & 46 & 164 & 7 & 230 \\
\hline & $\gamma$ & Enterobacteriales & Salmonella typhimurium & & GTAG & GCCBGRT & $A A$ & GGC n3-4 GCC & GCC & AYCVGGC & 134 & 18 & 65 & - & 6 \\
\hline & & & Klebsiella pneumoniae & & GTAG & SCCSGRT & $\mathrm{AA}$ & GGCG $n 3-4$ CGCC & GCC & AYCSGGS & 93 & 2 & 15 & 14 & 44 \\
\hline & & & Escherichia coli & & GTAG & GYCKGAT & AA & GRCGY n2-6 RCGYC & GC & ATCMGRC & 84 & 19 & 88 & 1 & 177 \\
\hline & & & Shigella flexneri & & GTAG & GYCKGAT & $\mathrm{AA}$ & & GC & ATCMGRC & 81 & 19 & 71 & 1 & 33 \\
\hline & $\gamma$ & Alteromonadales & Psychromonas ingrahamii & 4 & GTAG & GGTGCAT & & TCT & & ATGCACC & 18 & 1 & - & 2 & 28 \\
\hline & $\gamma$ & Chromatiales & Thioalkalivibrio HL-EbGR7 & & GTAG & GTCGGSCT & & $\mathrm{TC}$ & & AGSCCGAC & 10 & 62 & 2 & - & 14 \\
\hline & $\beta$ & & Neisseria meningitidis & 5 & GTAG & GTCGGATWC & & TY & & GWATCCGAC & 5 & 5 & - & - & - \\
\hline & s & Neisseriales & Neisseria gonorrhoeae & & GTAG & GTCGGATWC & & TY & & GWATCCGAC & 2 & 7 & - & - & - \\
\hline & & & & 6 & GTAG & WGCCGGCC & & GCT & & GGCCGGCW & 112 & 105 & 7 & 15 & 364 \\
\hline & & & Stenotrophomonas maltophilia & 7 & GTAG & MGYCGASY & & $\mathrm{n} 2-4$ & & RSTCGRCK & 70 & 84 & - & 7 & 78 \\
\hline & $\gamma$ & Xantnomonadales & Stenotropnomonas matopnita & 8 & GTAG & AKCCACGC & & CAY & & GCGTGGMT & 74 & 16 & 12 & 13 & 92 \\
\hline & & & & 9 & GTRG & GTGYSRACC & & (G) $\mathrm{TT}$ & & GGTYSRCAC & 66 & 22 & 6 & 19 & 180 \\
\hline & $\gamma$ & Alteromonadales & Idiomarina loihiensis & 10 & GTAG & CCTGACRT & & TY & & AYGTCAGG & 20 & 13 & - & 2 & 6 \\
\hline & $\gamma$ & Methylococcales & Methylomonas methanica & & GTAG & GGGCGAAT & & TY & & ATTCGCCC & 5 & 6 & - & - & - \\
\hline & $\delta$ & Desulfuromonadales & Geobacter uraniireducens & 11 & $\begin{array}{l}\text { GTAG } \\
\text { GTAG }\end{array}$ & $\begin{array}{l}\text { GGGCGGGG } \\
\text { GGGCGCAT }\end{array}$ & & $\begin{array}{l}\text { TYY } \\
\text { KGC }\end{array}$ & & $\begin{array}{l}\text { CCCCGCCC } \\
\text { ATGCGCCC }\end{array}$ & 29 & 17 & - & 1 & 3 \\
\hline Cyanobacteria & & Nostocales & Anabaena variabilis & 12 & GTAG & TSAGRACT & & TY & & AGTYCTSA & 27 & 12 & - & - & - \\
\hline & & & 「Actinobacillus succinogenes & & GTAG & GSYGGGCW & & TGCY & & WGCCCRSC & 10 & 7 & - & 1 & 3 \\
\hline & & Pasteurellales & Haemophilus influenzae & 13 & GTAG & GGTGGGCT & & $\mathrm{TY}$ & & AGCCCACC & 1 & 6 & - & 1 & 4 \\
\hline & 8 & Pseudomonadales & Pseudomonas mendocina & & GTAG & GGTGGGCT & & TY & & AGCCCACC & 22 & 2 & 1 & - & - \\
\hline Proteobacteria & & Alteromonadales & Ferrimonas balearica & & GTAG & CGTGGGCY & & $\operatorname{TnY}$ & & RGCCCACG & 11 & 1 & 3 & 1 & 52 \\
\hline & $\alpha$ & & Rhodopseudomonas palustris & & GTAG & GGTGGGCA & $\mathrm{AA}$ & GC GnA GC & G & TGCCCACC & 9 & 8 & - & - & 6 \\
\hline & $a$ & Rhizobiales & Bradyrhizobium ORS278 & 14 & GTAG & GGTGGGCA & $\mathrm{AA}$ & GGCGC $\mathrm{n} 3-4$ GCGCC & G & TGCCCACC & 66 & 36 & 1 & - & 9 \\
\hline Planctomycetes & & Planctomycetales & Planctomyces limnophilus & & GTAG & GGTGGGTT & & AAGGCTYTGCGC & & AACCCACC & 20 & 2 & - & 1 & - \\
\hline Flanctomycetes & & Pranctomycetales & Planctomyces brasiliensis & & GTAG & GCTGGGTTA & & GCCGRHAGGCG & & TAACCCAGC & 18 & 4 & - & - & - \\
\hline Cyanobacteria & & Nostocales & Anabaena variabilis & & GTAG & GTTGGGT & & GGA $n 6-9$ RAA & & ACCCAAC & 13 & 15 & - & - & 3 \\
\hline Cyanonacteria & & Chroococcales & Cyanothece PCC 7424 & 15 & GTAG & GTTGGGTT & $\mathrm{GA}$ & n5-8 & GA & AACCCAAC & 51 & 10 & - & - & 53 \\
\hline & $\delta$ & Desulfobacterales & Desulfatibacillum alkenivorans & & GTAG & GTTGGGTT & $\mathrm{GA}$ & GCTTGC & GA & AACCCAAC & 52 & - & - & - & - \\
\hline & $\gamma$ & Legionellales & Coxiella burnetii & & GTAG & GTTGGGCT & GA & GCTTGC & GA & AGCCCAAC & 9 & 12 & - & - & - \\
\hline Proteobacteria & $\beta$ & Neisseriales & Chromobacterium violaceum & & GTAG & GTTGGGCT & GA & GCTTGC & GA & AGCCCAAC & 4 & 1 & - & - & 11 \\
\hline & & Sulfurovum & Sulfurovum NBC37-1 & & GTAG & GGTGTGC & & AATW & & GCACACC & 8 & 9 & - & - & 15 \\
\hline Cyanobacteria & & Nostocales & Anabaena variabilis & 16 & GTAG & GGTGGGC & & AWT & & GCCCACC & 78 & 33 & - & - & 3 \\
\hline Cyanobacteria & & Chroococcales & Cyanothece PCC 7424 & & GTAG & GGTGGGC & & AHY & & GCCCACC & 201 & 3 & - & 8 & 27 \\
\hline & & & & 17 & GTAG & CCGGGCTC & & GTT & & GAGCCCGG & 75 & 38 & - & - & - \\
\hline Verrucomicrobia & & Opitutales & Opitutus terrae & 18 & GTAG & GGCYGSCG & & YTnRC & & CGSCRGCC & 34 & 53 & 1 & 1 & 3 \\
\hline & & & & 19 & GTGG & CAYGGGCG & & TCY & & CGCCCRTG & 104 & 61 & 22 & 3 & 19 \\
\hline & & & & 20 & GTAG & CWCGGYGG & & $\mathrm{T}(\mathrm{C}) \mathrm{CY}$ & & CCRCCGWG & 25 & 5 & 3 & 4 & 28 \\
\hline Planctomycetes & & Planctomycetales & Rhodopirellula baltica & 21 & GTAG & CYGgATTC & & $\operatorname{GCCA}(\mathrm{A})$ & & GAATtCRG & 24 & 13 & - & 8 & 72 \\
\hline & & & & 22 & GTMG & CCGACKGAG & & $\mathrm{GCC}$ & & CTCMGTCGG & 14 & 6 & 1 & 1 & - \\
\hline & & Thermomicrobiales & Thermomicrobium roseum & & GTAG & GGGYSAGGC & & GST & & GCCTSRCCC & 4 & 2 & 4 & - & 86 \\
\hline Chloroflexi & & Chloroflexales & Chloroflexus aggregans & 23 & GTMG & GGGCRMSSC & & Gnn & & GSSKYGCCC & 15 & 1 & - & 1 & 190 \\
\hline & & & Roseiflexus castenholzii & & GTAG & GGGCRSGBC & & $\mathrm{nnn}$ & & GVCSYGCCC & 34 & - & - & 6 & 63 \\
\hline & & & $\lceil$ Azotobacter vinelandii & & $\begin{array}{l}\text { GTAG } \\
\text { GTAG }\end{array}$ & $\begin{array}{l}\text { GGTGGAAAAC } \\
\text { GGTGGAAAAC }\end{array}$ & $\begin{array}{l}\text { CSGC } \\
\text { CBVSG }\end{array}$ & $\begin{array}{l}\text { GCA } \\
\text { GCA }\end{array}$ & $\begin{array}{l}\text { GCS- } \\
\text { GSBV- }\end{array}$ & $\begin{array}{l}\text { - TTTTCCACC } \\
\text { - TTTTCCACC }\end{array}$ & 31 & 8 & 1 & 1 & 4 \\
\hline Proteobacteria & $\gamma$ & Pseudomonadales & Pseudomonas stutzeri & 24 & GTAG & GGTGGAAAAI & NSGC & GMA & GCS - & -ТTTTCCACC & 37 & 1 & - & - & - \\
\hline & & & Pseudomonas aeruginosa & & GTAG & GGCGaATAA & CSSC & TY, Ann & GSSG & GTTATCCGCC & 32 & 7 & 8 & 1 & 98 \\
\hline
\end{tabular}

Figure 1 (See legend on next page.) 
(See figure on previous page.)

Figure 1 Families of GTAG repeats. The consensus sequences of GTAG-1 to GTAG-24 repeat families are reported. Families present in more than one species are boxed. Only the species, order and phyla are indicated (alpha to epsilon refer to Proteobacteria subdivisions). The complete names of the strains analyzed, and the NCBI accession numbers of the genomes are in Additional file 6. Loop sequences common to GTAG-3 and GTAG-14 elements from different species are boxed. Residues not present in all family members are in parentheses. Complementary nucleotide changes are indicated according to the NC-IUB codes (R=A,G; Y=C,T; $K=G, T ; M=A, C ; S=G, C ; W=A, T ; B=C, G, T ; H=A, C, T ; V=A, C, G)$. Non complementary stem residues are in lowercase letters. Gray numbers to the right refer to single elements (S), dimers (D: HH, $T \mathrm{~T}$ or $H T$ types; see text) or grouped elements $(\mathrm{G})$ in each family. Elements featuring alternative stem and loop sequences in G. uraniireducens GTA-11 and A. vinelandii GTAG-24 have been separately reported, but counted together (boxed gray numbers).

respectively. GTAG families 6 to 9 include all the $S$. maltophilia repeats previously called SMAGs [9]. Different REP families coexist also in A. vinelandii, C. burnetii, R. palustris, Bradyrhizobium sp. ORS278, A. variabilis, Cyanothece sp. PCC 7424, O. terrae, $R$. baltica. In contrast, different REPs reside in the two sequenced isolates of the Thioalkalivibrio genus Thioalkalivibrio sp. K90mix (GTAG-1 elements) and Thioalkalivibrio sp HL-EbGR7 (GTAG-5 elements).

Elements in Figure 1 are diagrammed in a modular fashion, to facilitate data presentation. In complex stemloop structures, as those featured by $E$. coli REPs, some complementary bases are viewed as part of the loop region, rather than of bulged stems. Elements assigned to different families have different stem or loop sequences, or both. The terminal GTAG motif, conserved in $>90 \%$ of the members of most repeat families, is variously degenerated in second and third position (GYAG, GYRG, GTRG, GTMG) in some families, and mutated to GTGG in the majority of $O$. terrae GTAG-20 elements. Most stems measure 6-9 bp. GTAG-1 repeats in Thauera sp. MZ1T have shorter stems (5 bp), all GTAG-24 repeats long (12-13 bp) stems. In the latter, complementarity is interrupted by mismatches in $P$. aeruginosa elements (unpaired GA residues in fifth position in all), $1 \mathrm{bp}$ bulges due to the presence/absence of residues in tenth position in GTAG-24 repeats in other species.

Most families can be subdivided into sub-families made by units which feature alternative complementary stem residues, as denoted by the NC-IUB code in Figure 1. GT pairing of stem residues was often observed, suggesting that many GTAG repeats may be transcribed and function as RNA elements. GTAG-1 and GTAG-2 markedly differ from all other repeats as they feature dinucleotides not involved in base pairing between the SLS region and the GTAG terminus, and conserved 3 bp motifs at the opposite side (Figure 1).

Loops come in a few main formats. Most loops are very short, and many fit the consensus TY or CMA. Minimal size loops (2-4 bp) are compatible with the formation of RNA hairpins [31]. Some loops, in contrast, have a complex structure. In all GTAG-3 elements but those found in $P$. mendocina, non complementary diand trinucleotides separate stem and loop sequences.
The simplest loops are featured by $C$. burnetii, $C$. sakazaki and Rhizobial elements, and consist of 2-4 bp regions flanked by GC residues. In other GTAG-3 families, loops with complementary GC/GC, GGC/GCC, and GRCG/CGYC termini coexist (see boxed sequences in Figure 1). The inner regions of the GRCG/CGYC loops are self complementary, and up to 6-7 bp paired regions can be formed. The relative abundance of loop types varies among GTAG-3 elements in different species. Long loops predominate among $E$. coli and $S$. flexneri elements, but are missing in E. cloacae. In contrast, units with GGC/GCC loops are missing in E. coli and S. flexneri, but represent more than $50 \%$ of the GTAG-3 elements in $K$. pneumoniae.

GTAG-14 repeats feature loops exhibiting a similar organization, and two and three major loop variants with different GC-rich termini were identified in $R$. palustris and Bradyrhizobium, respectively (Figure 1). The inner region of the GCGG/CCGC type loops, which have been found only in Bradyrhizobium elements, is made by complementary residues, and may measure up to $27 \mathrm{bp}$. Large loops (9-15 bp) are a feature of GTAG-15 elements. These loops are partly related in sequence and have the same termini of GTAG-3 and GTGA-14 repeat loops, but complementary bases are missing.

GTAG repeats may be found as single units, but many are associated and form characteristic structures. In several families, repeats are predominantly associated as dimers. Elements are next to each other (1-5 bp distance) in some dimers, but are located 20-100 bp apart in most. The relative orientation of partners determines the formation of three types of dimers. Dimers carrying GTAG termini outside or inside are referred as $\mathrm{HH}$ (head-head), and TT (tail-tail), respectively, those made by tandemly arranged repeats as HT (head-tail). Head and tail refer to the REP body and the terminal GTAG motif, respectively (see also ref. [9]). Some elements are grouped, and groups may include singletons as dimers arranged in different configurations. The smallest groups are represented by trimers, which can be viewed as singletons next to dimers of different types. Large REP clusters have a variable composition. Most include singletons or dimers reiterated in tandem, along with segments of flanking DNA of variable length. The number of singletons, dimers and grouped 
elements, vary extensively among GTAG families (Figure 1). Single elements predominate in families 14,16 and 24 respectively found in D. alkenivorans, Cyanothece sp. 7424 and $P$. stutzeri. In contrast GTAG-1 families in P. syringae, $X$. campestris and Thauera sp. Mz1T, the GTAG-3 family in C. sakazaki, and all GTAG-23 families are largely made by clustered elements. $\mathrm{HH}$ is the privileged type of dimer in most families, but TT dimers outnumber $\mathrm{HH}$ dimers in families 1, 3, 19 and 24. HT dimers are absent, or underrepresented, in most genomes.

T. roseum features two chromosomes, and GTAG-23 elements are distributed in both (Additional file 1).

\section{CGTC families}

CGTC elements are more similar to each other than GTAG elements, and have been assigned to only four families (Figure 2). Differences in sequence and overall structure of the main sequence types are ready to perceive by looking at the all families alignment at the bottom of Figure 2. The terminal CGTC motif is changed to TGTC or CCTC in many repeats. Stems measure 8 (families 1 and 2) or 9 bp (families 3 and 4), and almost invariably feature complementary AT residues in first and second position. Loops measure 4 (family 1) or 5 bp (families 2 to 4 ), and most fit a few major sequence types. Loops of different length and composition are found in Bradyrizobium CGTC-1, and $K$. olearia and $M$. prima CGTC-3 REPs. All CGTC elements end, similarly to GTAG-1 and GTAG-2 repeats, with short unpaired "tails", most of which fit the consensus CCA.

CGTC repeats have been found in microorganisms belonging to 5 phyla. Most reside in alpha-Proteobacteria, and CGTC REP families have been found in species of all the orders in which the alpha subdivision diverged [32]. The obligate bacterial predator Micavibrio aeruginosavorus, which hosts a family of CGTC-1 repeats, has been placed by phylogenetic analyses as a deep branch lineage within the alpha-Proteobacteria, and forms a sister clade to the Rhodospirillales order, that is otherwise distinct from the major alpha-Proteobacterial groups currently recognized [33]. Different CGTC REP families coexist in S. chlorophenolicum, S. wittichii, Bradyrhizobium and $R$. conorii (Figure 2).

Five of the species listed in Figure 2 (S. chlorophenolicum, A. tumefaciens, A. lipoferum, C. taiwanensis and $S$. meliloti) have either two chromosomes, or one chromosome and one or more megaplasmids. The total number of repeat types in each organism is reported in Figure 2. The number of repeats in chromosomes and megaplasmids is reported in Additional file 1.

CGTC repeats are as heterogeneous as GTAG repeats, as illustrated by the extensive use of the IUB code in Figure 2, needed because several families include subsets made by units having different stem, loop or tail sequences. CGTC elements are predominantly organized as $\mathrm{HH}$ dimers. TT dimers are rare, HT dimers negligible. Grouped elements are also rare, but it is worth noting that most of the elements found in Neisseriae and Wolbachia are organized in large clusters.

Some repeats correspond to described sequences. CGTC-1 elements in Neisseriae correspond to the dRS3 repeats [34], CGTC-1 and CGTC-4 elements in R. conorii to RPE-6 and RPE-4 repeats [13], respectively. In contrast, the CGTC-4 elements identified in the genomes of the Wollbachia endosymbionts of $D$. simulans and $D$. melanogaster are unrelated to the palindromic WPE repeats identified in the Wolbachia endosymbiont of Brugia malayi [35].

\section{Association of GTAG and CGTC to other repeats}

The diversity of flanking DNA suggests that most REPs are not associated to other sequence repeats. We have not investigated this issue in detail, because out of the scope of this paper. Yet, it is worth mentioning that members of a few REP families repeats are regularly associated to similar DNA tracts. Many A. variabilis GTAG-15 dimers are inserted within long palindromic sequences fitting the consensus TATAGGAnTnnnATTTGATTnnTGAAA .TTTCAnnAATCAAATnnnAnTCCTATA (capital letters denote complementary bases, dots GTAG-15 dimers). T. roseum GTAG-23 elements are inserted within small palindromes fitting the consensus CCGSSCC (n3, 4) GGSSCGG, all the $H$. neapolitanus CGTC-1 dimers within 41 bp palindromic sequences, fitting the consensus GGGaaGCTT-GAAAaACC••attcacgGGTaTTTCgAAGCgCCC (letters and dots are as above). Target palindromes unlinked to REP sequences were not found in $A$. variabilis as in $H$. neapolitanus DNA. In contrast, hundreds copies of the GTAG-23 target occur in the GC-rich T. roseum genome. Many of the Neisseria CGTC-1 elements clustered in large mosaic intergenic regions are interleaved with members of different repeat families [36].

\section{Variations of GTAG and CGTC families}

The organization of abundant REP families was analyzed in genomes of the same or related species. We monitored the relative abundance of the predominant sequence types (STs), as changes in the distribution of singletons, dimers and grouped elements. Data on species containing one or more REP families are reported in Figure 3. No significative variations were found in families of repeats residing in $P$. aeruginosa, $H$. infuenzae, S. maltophilia, N. meningitidis, N. gonhorroeae, C. burnetii.

Changes in the organization of specific families among strains and/or species are discussed below.

\section{Pseudomonas REPs}

The compared strains of $P$. syringae [37] P. fluorescens [38] and P. putida [39] represent major phylogenetic 


\section{CGTC families}

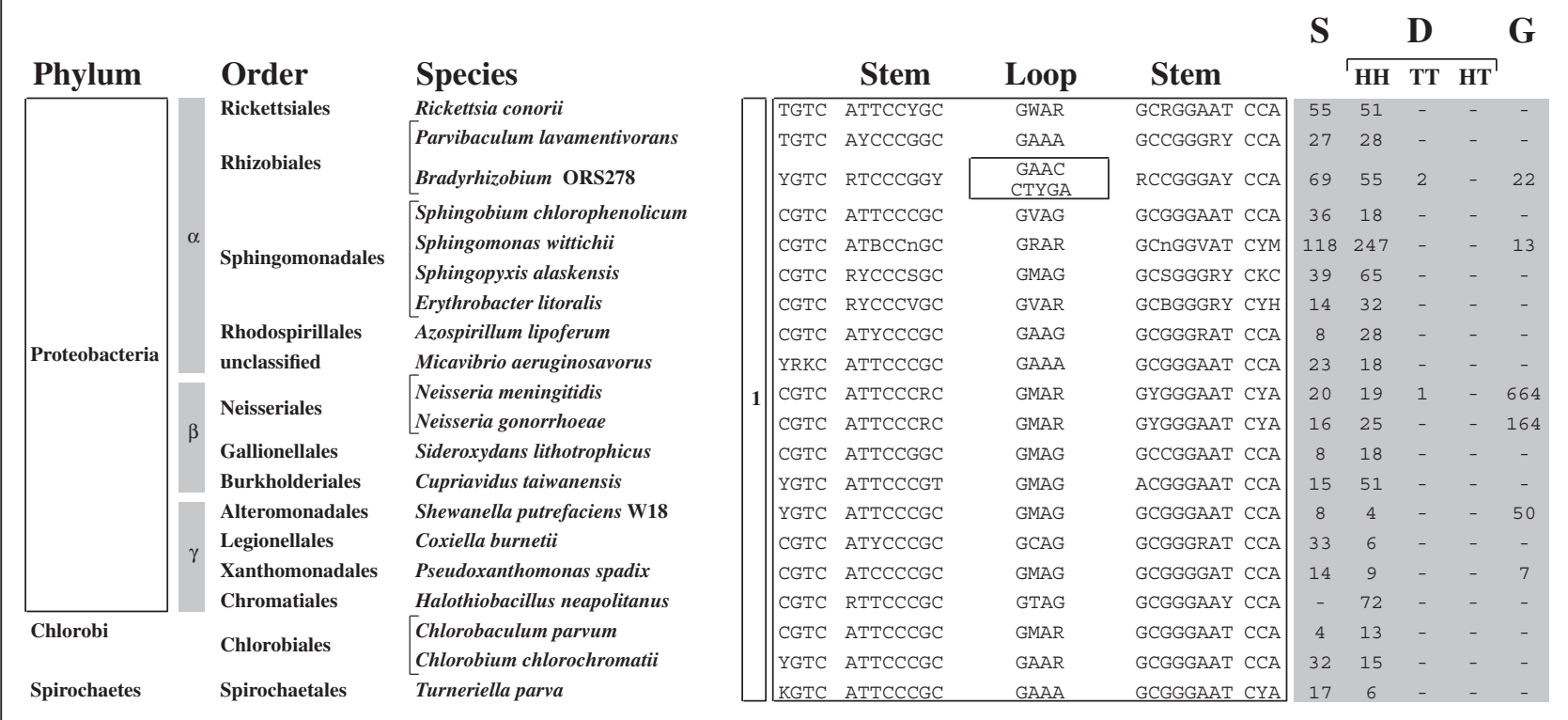

\begin{tabular}{|c|c|c|c|}
\hline \multirow{5}{*}{ Proteobacteria } & $\alpha$ & Sphingomonadales & $\begin{array}{l}\text { Sphingobium chlorophenolicum } \\
\text { Sphingomonas wittichii } \\
\text { Novosphingobium aromaticivorans }\end{array}$ \\
\hline & & Alteromonadales & Shewanella woodyi \\
\hline & $\gamma$ & Oceanospirillales & Kangiella koreensis \\
\hline & & Thiotrichales & Francisella tularensis \\
\hline & $\varepsilon$ & Sulfurovum & Sulfurovum NBC37-1 \\
\hline Bacteroidetes & & Flavobacteriales & Gramella forsetii \\
\hline Thermotogae & & Thermotogales & {$\left[\begin{array}{l}\text { Kosmotoga olearia } \\
\text { Mesotoga prima }\end{array}\right.$} \\
\hline
\end{tabular}

\begin{tabular}{|c|c|c|c|c|c|c|c|c|}
\hline CGTC & ATGCTGAA & CTTGT & TTCAGCAT CCA & 22 & 9 & - & - & - \\
\hline CGTC & ATSCYGRA & CTYGR & TYCRGSAT CCA & 24 & 26 & - & - & - \\
\hline CGTC & AYSCTGAA & CTTGT & TTCAGSRT CCA & 3 & 24 & - & - & 3 \\
\hline YGTC & ATCCYGRR & СTTGT & YYCRGGAT CCA & 22 & - & - & - & - \\
\hline TGTC & ATCCTGAA & CTKGA & TTCAGGAT CTG & 11 & - & 5 & - & - \\
\hline CGTC & ATSCTGAA & YTTRT & TTCAGYAT CTC & 4 & 4 & - & - & - \\
\hline YGTC & ATYCTGAA & CTGYT & TTCAGRAT CYC & 9 & 5 & - & - & - \\
\hline CGTC & AHBCTGAA & YTTRT & TTCAGVDT CTB & 67 & 39 & 2 & 1 & - \\
\hline YGTC & АTYCTGGA & $\begin{array}{c}\text { CTYGA } \\
\text { AATSTTYWA }\end{array}$ & TCCAGRAT CTK & 37 & 21 & - & - & 40 \\
\hline YGTC & ATBCTGAA & $\begin{array}{c}\text { CTYGW } \\
\text { (R) RHGMTCC }\end{array}$ & TTCAGGAT CTM & 63 & 4 & 1 & 2 & - \\
\hline
\end{tabular}

\begin{tabular}{|l|l|l|l}
\hline & Rhizobiales & {$\left[\begin{array}{l}\text { Xanthobacter autotrophicus } \\
\text { Starkeya novella } \\
\text { Oligotropha carboxidovorans } \\
\text { Bradyrhizobium ORS278 } \\
\text { Rhodopseudomonas palustris } \\
\text { Agrobacterium tumefaciens } \\
\text { Rhizobium } \text { etli } \\
\text { Sinhorizobium melitoti } \\
\text { Pelagibacterium halotolerans } \\
\text { Mesorhizobium } \text { BNC1 }\end{array}\right.$} \\
& Caulobacterales \\
Rickettsiales & {$\left[\begin{array}{l}\text { Rickettsia conorii } \\
\text { Wolbachia } \text { wi }\end{array}\right.$}
\end{tabular}

\begin{tabular}{llll|lcccc|}
\hline CGTC & ATGSCCGGG & CTTGW & CCCGGSCAT CCA & 29 & 37 & - & - & - \\
CSTC & ATSSCCGGG & CTTGR & CCCGGSSAT CCA & 80 & 102 & 1 & 1 & 25 \\
YGTC & ATGSCCGGV & YTTRW & BCCGGSCAT CCA & 56 & 45 & - & - & 3 \\
CGTC & ATGSCCGGG & CTTGW & CCCGGSCAT CCA & 46 & 32 & - & - & - \\
CGTC & ATGSGCGGG & CTYGW & CCCGCSCAT CCA & 49 & 78 & 2 & 1 & 7 \\
CSTC & ATYCYYGKG & CTYGT & CMCRRGRAT CYR & 29 & 78 & - & - & - \\
CSTC & ATYCYYGKG & CYYGT & CMCRRGRAT CYR & 25 & 21 & - & - & 4 \\
CCTC & ATYCCTGTG & CYYGT & CMCAGGRAT CCA & 58 & 9 & - & - & 31 \\
CGTC & RTCCTCGGG & CTYGA & CCCGGGGAY CYG & 9 & 9 & - & - & 32 \\
CGTC & ATCCTCGGG & CTTGA & CCCGAGGAT CCA & 3 & - & 8 & - & 4 \\
\hline
\end{tabular}

4 \begin{tabular}{llll|ccccc|} 
CGTC & ATCCCGCGC & YKYRT & GCGCGGGAT CCA & 2 & 19 & - & - & - \\
TGTC & ATMCCGYGR & CTTGA & YCRCGGKAT CYA & 40 & 13 & - & - & - \\
CGTC & ATMCCGCKA & YTYRT & TMGCGGKAT CDM & 40 & 30 & 1 & 1 & 140
\end{tabular}

$\begin{array}{llllll}\text { CGTC-1 } & \text { YGTC } & \text { RTYCCSGC } & \text { GVAR } & \text { GCSGGRAY } & \text { CnA } \\ \text { CGTC-2 } & \text { YGTC } & \text { AYBCYGRA } & \text { YTYGD } & \text { TTCRGVRT } & \text { CYn } \\ \text { CGTC-3 } & \text { CGTC } & \text { ATBSBYGKG } & \text { YTYGW } & \text { CMCRRSVAT CYR } \\ \text { CGTC-4 } & \text { CGTC } & \text { ATMCCGYKV } & \text { YKYRW } & \text { BMRCGGKAT CnM }\end{array}$

Figure 2 Families of CGTC repeats. The consensus sequences of CGTC-1 to CGTC-4 repeat families are reported. Data are presented as in Figure 1. Differences among the four repeat types are highlighted by the all families alignment at the bottom. 


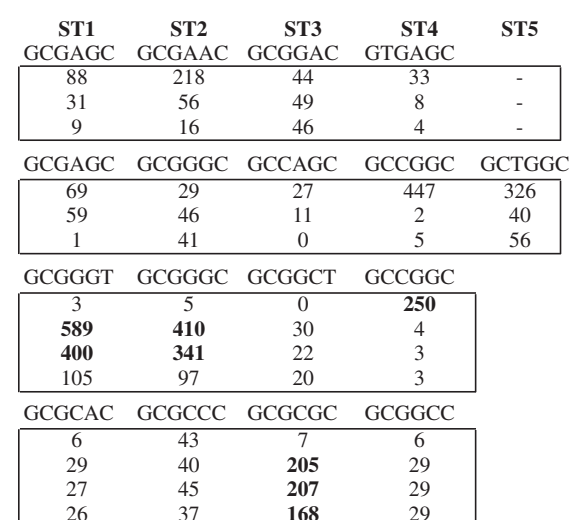

GCCGGAT GTCTGAT GCCGGGT GCCCGGT

\begin{tabular}{|c|c|}
\hline GTAG-3 & $\begin{array}{l}\text { S. enterica ser Typhi Ty2 } \\
\text { S. enterica ser Typhimurium LT2 } \\
\text { S. enterica ser Paratyphi A ATCC } 9150 \\
\text { S. enterica ser Paratyphi C RKS4594 } \\
\text { S. enterica ser Choleraesuis } \\
\text { S. enterica ser Dublin } \\
\text { S. enterica ser Enteritidis } \\
\\
\text { E. coli } K-12 \text { MG1655 } \\
\text { E. coli } K-12 \text { W3110 } \\
\text { E. coli O157:H7 EC4115 (EHEC) } \\
\text { E. coli O157:H7 EDL933 (EHEC) } \\
\text { E. coli O6:K2:H1 CFT073(UPEC) } \\
\text { E. coli O6:K15:H31 } 536 \text { (UPEC) } \\
\text { E. coli SMS-3-5 (environmental) } \\
\text { E. coli O152:H28 SE11 (commensal) } \\
\text { E. coli O7:K1 IAI39 (ExPEC) } \\
\text { E. coli O17:K52:H18 UMNO26 (ExPEC) }\end{array}$ \\
\hline Cyanobacteria & $\begin{array}{l}\text { A. variabilis } \\
\text { N. punctiforme PCC } 73102 \\
\text { N. punctiforme PCC } 7120 \\
\text { Cyanothece sp. PCC } 7424 \\
\text { Cyanothece sp. PCC } 7425 \\
\text { Cyanothece sp. PCC } 7822 \\
\text { Cyanothece sp. PCC } 8801 \\
\text { Cyanothece sp. PCC } 8802 \\
\text { Cyanothece sp. } 51142\end{array}$ \\
\hline Bradyrhizobium & $\begin{array}{l}\text { Bradyrhizobium sp. ORS278 } \\
\text { Bradyrhizobium japonicum } \\
\text { Bradyrhizobium sp. BTAi1 }\end{array}$ \\
\hline R. palustris & $\begin{array}{l}\text { R. palustris CGA009 } \\
\text { R. palustris HaA2 } \\
\text { R. palustris BisB } 18 \\
\text { R. palustris BisB5 } \\
\text { R. palustris BisA53 } \\
\text { R. palustris TIE-1 } \\
\text { R. palustris DX-1 }\end{array}$ \\
\hline C. burnetii & $\begin{array}{l}\text { C. burnetii } R S A 493 \\
\text { C. burnetii } G(Q 212) \\
\text { C. burnetii } K(Q 154) \\
\text { C. burnetii Dugway (5J108-111) }\end{array}$ \\
\hline P. mendocina & $\begin{array}{l}\text { P. mendocina ymp } \\
\text { P. mendocina } N K-01\end{array}$ \\
\hline Rickettsiae & $\begin{array}{l}\text { R. conorii } \\
\text { R. akari } \\
\text { R. bellii OSU_85 } \\
\text { R. canadensis } \\
\text { R. prowazekii } \\
\text { R. typhi } \\
\text { R. felis } \\
\text { R. massimiliae } \\
\text { R. peacockii } \\
\text { R. rickettsii Iowa }\end{array}$ \\
\hline
\end{tabular}

P. syringae $B 728$ a

P. syringae $D C 3000$

P. fluorescens pf05

P. fluorescens $\mathrm{pfO} 1$

P. fluorescens SWB25

GTAG-1

P. putida W619

P. putida F1
P. putida $K T 2440$

P. putida GB1

X. campestris $85-10$

$X$. campestris $B 100$

X. campestris 8004

enterica ser Paratyphi C RKS4594

S. enterica ser Choleraesui.

enterica ser Dublin

E. coli $K-12$ MG1655

O6:K2:H1 CFT073(UPEC)

. coli OI 25 :H28 SE11 (commensal)

coli O7:K1 IA139 (EXPEC)

. punctiforme PCC 73102

sp. PCC 8801

sp. ORS278

palustris $C G A 009$

palustris BisB 18

palustris $D X-1$

R. rickettsii Iowa
X. campestris ATCC-33913

\begin{tabular}{|llll|}
\hline 215 & 54 & 16 & 56 \\
209 & 60 & 16 & 51 \\
227 & 62 & 18 & 55 \\
218 & 59 & 20 & 56 \\
216 & 59 & 19 & 53 \\
222 & 52 & 16 & 52 \\
215 & 54 & 16 & 54 \\
\hline
\end{tabular}

GCCGGAT GCCTGAT GTCGGAT

\begin{tabular}{|ccc|}
\hline 206 & 196 & 58 \\
206 & 195 & 57 \\
139 & 132 & 46 \\
135 & 132 & 48 \\
150 & 136 & 52 \\
137 & 141 & 53 \\
102 & 91 & 24 \\
134 & 95 & 33 \\
82 & 80 & 24 \\
133 & 130 & 47 \\
\hline
\end{tabular}

GTAG-12 GTAG-15 GTAG-16

\begin{tabular}{|ccc|}
\hline 39 & 31 & 102 \\
24 & 25 & 3 \\
20 & 27 & 77 \\
- & 114 & 239 \\
- & - & - \\
- & 10 & 17 \\
- & 12 & 122 \\
- & 14 & 125 \\
- & 25 & 40 \\
\hline GTAG-3 & GTAG-14 & CGTC-1
\end{tabular}

\begin{tabular}{|cccc|} 
GTAG-3 & GTAG-14 & CGTC-1 & CGTC-3 \\
\hline 160 & 0 & 126 & 86 \\
26 & 0 & 60 & 33 \\
39 & 0 & 25 & 82 \\
11 & - & - & 111 \\
- & 20 & - & 138 \\
118 & 31 & - & 76 \\
15 & 2 & - & 36 \\
12 & 28 & - & 197 \\
- & - & - & 108 \\
- & 3 & - & 82 \\
\hline
\end{tabular}

\begin{tabular}{|ccc|}
\hline GTAG-3 & GTAG-15 & CGTC-1 \\
\hline 206 & 32 & 40 \\
199 & 31 & 44 \\
194 & 39 & 41 \\
240 & 42 & 47 \\
\hline GTAG-1 & GTAG-4 & GTAG-13 \\
\hline 59 & 136 & 20 \\
\hline
\end{tabular}

\begin{tabular}{cc}
256 & 108 \\
\hline CGTC-1 & CGTC- \\
\hline 168 & 65 \\
126 & 71 \\
99 & 128 \\
56 & 28 \\
- & - \\
- & - \\
211 & 74 \\
181 & 61 \\
173 & 66 \\
160 & 66 \\
\hline
\end{tabular}

Figure 3 Strain variations of REP families. For GTAG-1 and GTAG-3 families, the relative abundance of major sequence types (ST) in the indicated strains are shown. For clarity, of each ST only left-hand, stem sequences are reported. Abundant sequence-subfamilies are highlighted. 
clades, adapted to specific lifestyles and environmental niches. The number of GTAG-1 repeats varied in the genomes examined over a 5-10 fold range, mostly for the expansion of specific repeat sub-populations. The $P$. putida F1 and KT2440 strains are overrun by ST1 and ST2 units, but have few ST4 units, which in contrast are predominant in the W619 strain (Figure 3). Similarly, the large sizes of the GTAG-1 families in P. fluorescence Pf-05 and P. syringae B728A genomes are correlated to the expansion of ST2 and ST4 units, respectively. Many of these repeats are reiterated in tandem, suggesting that amplification and clustering of REPs may be correlated processes.

\section{Enterobacterial REPs}

The number of GTAG-3 repeats was comparable in all the strains of Salmonella enterica analyzed, but varied over a twofold range among pathogenic, laboratory and environmental E. coli strains. The organization of GTAG3 repeats found in the known MG1655 E. coli strain is largely conserved in all the strains analyzed, and size changes of the various repeat families are not correlated to the expansion of specific STs, but rather to an increased number of dimers and clustered elements in MG1655 DNA.

\section{Bradyrhizobia REPs}

The organization of REP families was monitored in three strains of the genus Bradyrhizobium, and six strains of R. palustris. Bradyrhizobium sp. ORS278 and BTAi1 are photosynthetic bacteria, isolated from stem nodules of different Aeschynomene species, B. japonicum USDA110 is a non-photosynthetic rhizobium able to form root nodules on soybeans [40]. The relative abundance of GTAG-3, GTAG-14, CGTC-1 and CGTC-3 elements varied over a 8 -fold range among the three strains, each repeat peaking in one or two strains only (Figure 3). While comparable in size, GTAG-14 families in Bradyrhizobium sp. ORS278 and B. japonicum USDA110 significantly differ in their organization. Units with large GCGG/CCGC type loops (see Figure 1) are very few in B. japonicum DNA, but the number of $\mathrm{HH}$ dimers found in this species is much higher than in Bradyrhizobium sp. ORS278 (59 vs 38 dimers).

The size and the pattern of distribution of GTAG-3, GTAG-14, and CGTC-3 families in the six $R$. palustris strains analyzed does not match the hierarchical clustering resulting from the analysis of Pfam domains, according to which BisA53 and BisB18 strains cluster together, BisB5, HaA2, CGA009, and TIE-1 strains on a distinct branch, with CGA009 and TIE-1 on the same node [41]. GTAG-3 elements peak in BisB18, are 10-fold less abundant in other strains, and missing in TIE-1. CGTC-3 elements reside in all strains, but their abundance varied over a 5- fold range, moderately abundant families of GTAG-14 repeats in BisB18, BisA53 and HaA2 strains only.

\section{Cyanobacterial REPs}

GTAG-15 and GTAG-16 elements were monitored in three filamentous (Anabaena variabilis, Anabaena sp. strain PCC 7120, Nostoc punctiforme PCC 73102) and six unicellular cyanobacteria of the genus Cyanothece (51142, 7424, 7425, 7822, 8801 and 8802 strains) showing high genetic variation [42]. Both GTAG-15 and GTAG-16 elements peak in the 7424 strain, are 2-10 fold less abundant in other strains, and are missing in the 7425 strain. Curiously, the DNA of this strain has a GC content significantly higher than the DNAs of the other strains analyzed (49\% vs. 37-39\%; see ref. [42]). GTAG-12 repeats were detected in filamentous Cyanobacteria only, and are two times more abundant in $A$. variabilis than in Anabaena sp. strain PCC 7120 and Nostoc punctiforme PCC 73102.

\section{Rickettsial REPs}

CGTC-1 and CGTC-4 repeat families varied in size over a two-fold range in many species of the genus Rickettsia. The lowest number of repeats was found in $R$. canadensis. Neither CGTC-1 nor CGTC-4 elements were found in $R$. prowazeki and $R$. typhi, a result in line with literature data indicating that both species lack repetitive sequences [43].

\section{Organization of REP dimers}

GTAG as CGTC elements are frequently associated to form dimers. The relative abundance of REP dimers im most families is underestimated, as a consequence of both sequence variation and the insertion of DNA between dimer partners. In P. fluorescence, most GTAG-1 singletons are remnants of $\mathrm{HH}$ dimers [26], and this may hold true for more species upon closer inspection. The components of $\mathrm{HH}$ or TT dimers may fold separately, or form a single, large SLS $[9,44]$. Both $\mathrm{HH}$ and TT dimers can be further distinguished because made up by the same elements (homodimers), or elements which feature different stem and/or loop sequences (heterodimers). Further variation was observed in $S$. maltophilia, about $10 \%$ of dimers found in this microorganism being heterodimers formed by members of different GTAG families (hybrid dimers; the components of these dimers have been counted as singletons in Figure 1). The number of homodimers and heterodimers varies significantly among REP families. Most $\mathrm{HH}$ and TT GTAG-1 dimers in P. entomophila and P. putida are homodimers. In contrast, GTAG-3 dimers in Enterobacteriaceae are exclusively formed by elements with loops of different lengths, and $P$. aeruginosa GTAG-24 dimers by elements with different stems (see changes at stem residues 12 and 13 in Figure 1). Homodimers predominate 
among CGTC-1, heterodimers among CGTC-2 and CGTC-3 elements. Yet only heterodimers are formed by H. neapolitanus and C. taiwanensis CGTC-1 repeats, as only homodimers by $N$. aromatocivorans CGTC-2 and $A$. tumefaciens CGTC-3 repeats.

The preferential formation of heterodimers over homodimers in most CGTC and GTAG families has no obvious explanation. Dimers may form large DNA hairpins in single-stranded state or DNA cruciforms. These structures cause replication stalling, and in turn lead to genome instability, and need to be eliminated by specific enzymes during DNA replication [45]. The deletion frequency is significantly influenced by the stability of base pairing involving the first $16-20 \mathrm{bp}$ stem residues [46]. In E. coli secondary structures formed by IRs are removed by enzymes of the SbcCD complex, and the minimum duplex stem length necessary for cleavage lies between 8 and 16 bp [47]. These considerations suggest that heterodimers may be protected from enzymatic degradation and genome clearance. Large secondary structures formed by pairing of adjacent REPs may have functional relevance at the RNA level, and differences in the extent of base pairing between homodimers and heterodimers may determine whether the RNA hairpins formed are sensitive or resistant to cleavage by specific endoribonucleases $[17,19]$.

The distance between dimer partners is variable. Only 1-2 bp separate the partners of $O$. terrae GTAG-17 HH and GTAG-19 TT dimers. The same holds for Wolbachia CGTC-4 dimers, and in some both spacer and a few adjacent REP bases have been deleted. In most dimers, spacers vary in length from 20 to $100 \mathrm{bp}$. Some are largely conserved, others differ in sequence but have similar lengths, or differ both in sequence and size. As a rule of thumb, TT and $\mathrm{HH}$ dimers feature variable and conserved spacers, respectively. However, as illustrated in Figure 4, different spacer types may coexist in large dimer families. Several dimers carry spacers which feature either complementary ends, or small SLSs at one end. Two distinct SLSs are at the ends of the spacer in several A. tumefaciens CGTC-3 dimers (Figure 4). The presence of structured spacers immediately suggest that dimers may fold into stable hairpins.

It may be of interest noting how the relative abundance of different spacer types may vary among related species. P. putida GTAG-1 HH dimers have three types of spacers. Of these, only one is conserved in $P$. entomophila elements, and at lower abundance. The number of GTAG-1 TT dimers in the two species is comparable, but the relative amount of spacers with complementary ends is significantly different.

\section{Genome distribution of REP sequences}

Members of most of the REP families identified are spread throughout the genome. A noticeable exception is represented by $T$. roseum GTAG-23 elements, which are clustered in large blocks at few loci.

Most REPs are located in the intergenic space. Relative to the orientation of flanking ORFs, repeats may be located between either convergently (conv-REPs), or divergently (div-REPs), or unidirectionally (uni-REP) transcribed ORFs. In different REP-rich genomes the repeats are predominantly located between unidirectionally and convergently transcribed ORFs (Figure 5). This finding reinforces the notion that most REPs are transcribed, and may function as RNA sequences. The distances separating P. entomophila GTAG-1 and S. wittichi CGTC-1 elements from flanking ORFs are diagrammed in Figure 6. The pattern of interspersion of singletons and dimers, separately analyzed, is similar. In P. entomophila as in S. wittichi, most convREPs are next $(<20 \mathrm{bp})$ to the $3^{\prime}$ end of both flanking ORFs. Uni-REPs are also located close to the 3 ' end of upstream ORFs, but are at varying distances from downstream ORFs. This suggests that the fraction of readthrough transcripts spanning REPs, that may influence the expression of both flanking ORFs, may be limited. The pattern of interspersion of GTAG-1 and CGTC-1 elements and flanking ORFs did not vary in other REP-rich genomes analyzed (Additional file 2).

Members of several REP families are close to, or even overlap coding regions. The extent of contiguity is immediately illustrated by the finding that the termini of GTAG REPs often provide the opal stop codon (TAG) to flanking ORFs. In different species, a variable number of REPs are entirely located within ORFs. Target ORFs and REP-encoded amino acids are listed in Additional file 3, data are summarized in Figure 7 . In all the genomes examined, a plethora of regions, selected on the base of arbitrary length thresholds, have been annotated as ORFs, but encode short proteins plausibly all spurious. Therefore, REPs mapping within hypothetical proteins $<120$ amino acids have been not included in the pool of intragenic elements.

The highest number of intragenic GTAG and CGTC repeats were found in $O$. terrae and $R$. conorii, respectively (Figure 7A). Intragenic $R$. conorii repeats correspond to the described RPE-4 and RPE- 6 elements [13], and is worth recalling that other genes are interrupted in this species by longer palindromic insertions called RPE1 [14]. More than 50\% of the inserts are dimers or grouped repeats, which encode 20 to 30 amino acids. In some $O$. terrae and $R$. conorii ORFs, single elements and/ or dimers are inserted twice, at close or distant sites. Larger REP-encoded regions have been found in Thauera and $R$. conorii, where clusters of repeats encode 43 to 82 amino acids (Additional file 3). The remaining elements are variably located along ORFs. Slightly more than $10 \%$ of GTAG and CGTC repeats are at the end of the coding region, a higher number at the ORF NH2 terminus. Of 


\begin{tabular}{|c|c|c|c|c|c|}
\hline REP & Species & Dimer & $\%$ & bp & Features \\
\hline \multirow{6}{*}{ GTAG-1 } & \multirow{4}{*}{$P \cdot$ putida } & \multirow{3}{*}{ HH (88) } & 27 & 39 & TGCG . . CGCA \\
\hline & & & 15 & 58 & [AGGGCcgGCCCT] \\
\hline & & & 56 & 37 & [CACCSGCnnnGCSGGTG] \\
\hline & & TT (234) & 26 & $34-36$ & AGG. . . CCT \\
\hline & \multirow{2}{*}{ P. entomophila } & HH (282) & 81 & $36-40$ & [CACCSGCgtaGCSGGTG] \\
\hline & & TT (284) & 4 & $34-36$ & AGG . . CCT \\
\hline \multirow{2}{*}{ GTAG-3 } & \multirow{2}{*}{ E. coli } & $\mathrm{HH}(21)$ & 75 & $16-17$ & [GACGCtgn $1-2$ CGCGTC ] \\
\hline & & TT (90) & 61 & 35 & RHDDDYHYTGCAATWTATTGAATTTGCRBGHTTTT \\
\hline \multirow[t]{4}{*}{ GTAG-7 } & S. maltophilia & $\mathrm{HH}(83)$ & 39 & $34-36$ & [CGCGCgcaGCGCG] \\
\hline & \multirow{2}{*}{ R. conorii } & \multirow{2}{*}{ HH (51) } & 37 & $62-85$ & [AAACATATAAAAAn4 - 6TTTTTATAGTTT] \\
\hline & & & 27 & $36-92$ & [AAAGCgaGATaaATCgaGCTTT] \\
\hline & H. neapolitanus & $\mathrm{HH}(80)$ & 100 & 15 & GCGCCTTGATTTTTC \\
\hline \multirow[t]{5}{*}{ CGTC-1 } & \multirow{4}{*}{ S. wittichii } & \multirow{4}{*}{ HH (237) } & 11 & $41-44$ & TGTCT . AGACA \\
\hline & & & 24 & 36 & GGGAG . . CTCCC \\
\hline & & & 26 & $18-46$ & CCT ..... AGG \\
\hline & & & 27 & $39-40$ & CGGTC. . ATCCA \\
\hline & S. alaskensis & HH (64) & 67 & $23-24$ & GCC . . GGT \\
\hline \multirow{9}{*}{ CGTC-3 } & S. novella & HH (102) & 70 & $24-37$ & CGnCTT. . AAGnCG \\
\hline & Bradyrhizobium & HH (32) & 87 & $26-28$ & CGTCTT. . AAGACG \\
\hline & \multirow{2}{*}{ O. carboxidovorans } & \multirow{2}{*}{ HH (49) } & 53 & $24-30$ & CGTCTT. . AAGACG \\
\hline & & & 41 & $8-28$ & TC.......GA \\
\hline & \multirow{3}{*}{ R. palustris } & \multirow{3}{*}{ HH (78) } & 31 & $21-31$ & CGTCTT . . AAGACG \\
\hline & & & 27 & $12-44$ & TC......GA \\
\hline & & & 31 & $24-30$ & CG........ CG \\
\hline & \multirow[t]{2}{*}{ A. tumefaciens } & \multirow[t]{2}{*}{ HH (72) } & 40 & $56-59$ & $\begin{array}{l}\text { [GCCGACGcgCGTCtGC] } \\
\text { [CAGCCCAAGgaCTTGGGCTG] }\end{array}$ \\
\hline & & & 60 & 27 & AnCACGTnnCnTAAAATCRAnnSGTTG \\
\hline
\end{tabular}

Figure 4 Spacers in REP dimers. The organization of spacer sequences in abundant families of dimers is shown. The number of HH or $\Pi T$ dimers [in parentheses] and the relative abundance of the spacer variants are shown. Spacer features include complementary ends or SLSs (in brackets; complementary bases are in capital letters). The two SLSs in A. tumefaciens spacers are separated by 20-23 bp. The sequence of the E. coli $\pi$ dimer spacers is from reference [48].

these, many may be extragenic, since translation may initiate not at the predicted, but rather at downstream sites. As inferred by alignment to shorter homologous proteins encoded by either related species, or strains of the same species, most REPs located within the $5^{\prime}$ end of P. putida, C. koseri and S. maltophilia ORFs may be not codogenic, but rather function as post-transcriptional control elements. On the other hand, R. conorii proteins decorated by RPE-1 elements at the NH2 terminus are expressed in vivo [49]. Would we ignore all ORFs carrying
REPs in the NH2 terminus, the number of ORFs decorated by REPs is still high.

The encoded proteins belong to different categories, but many play a role in DNA synthesis and repair. Different species potentially encode REP-decorated proteins involved in nucleotide excision (excinuclease $\mathrm{ABC}$ complex proteins, UvrD/REP helicase, DNA polymerase I), or in homologous recombination repair (recBCD proteins; Figure 7B). The two uvrA genes found in O. terrae are both interrupted at different sites by dual REP inserts. REP- 

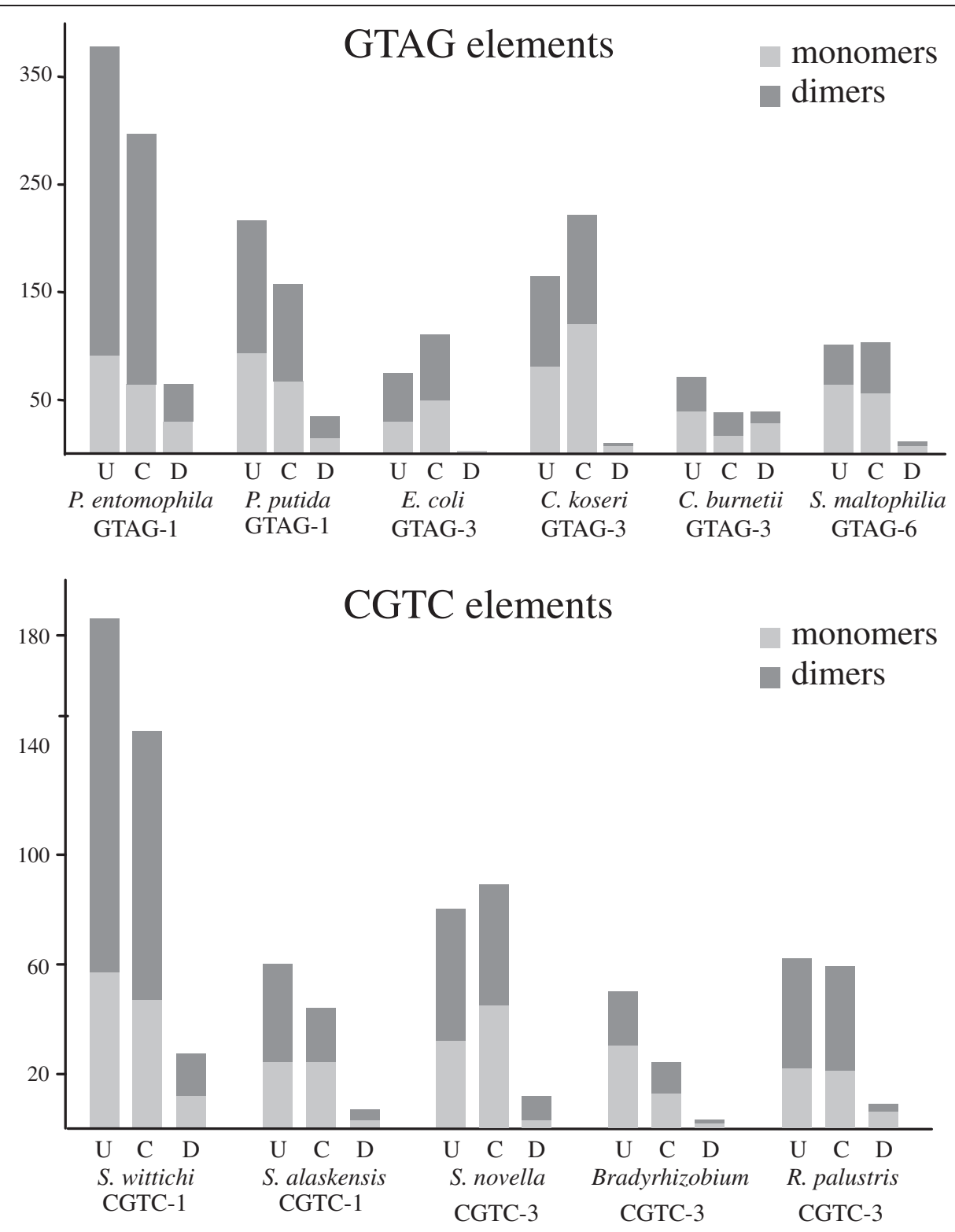

Figure 5 REPs and flanking ORFs. The number of single REPs and dimers located between convergently (conv-REPs; C), divergently (div-REPs; $D$ ), and unidirectionally (uni-REP; $U$ ) transcribed ORFs in different species is shown.

tagged proteins include the inducible, error prone DNA polymerases, encoded by DnaE2 genes [50]. In $R$. conorii, which lacks DnaE2, a REP element is inserted within the DnaE gene, which encodes the high-fidelity replicative polymerase (Figure 7B). Remarkably, some of the listed ORFs are the only coding sequences modified by REPs in a given species. REPs are also inserted in other genes involved in DNA repair, such DNA ligase in O. terrae, a DNA-photoreactivating enzyme in Thauera, as in genes encoding RNA binding proteins, such RNA helicases in $O$. terrae, tRNA synthetases in $X$. oryzae, E. lithoralis and $S$. alaskensis, tRNA pseudouridine synthase B subunit genes in S. maltophilia, E. lithoralis and S. alaskensis. Curiously in S. maltophilia, also the A subunit gene is interrupted by a REP (Additional file 3). In light of these findings, may be worth recall that the $R$. conorii tRNA pseudouridine synthase B subunit gene is interrupted by RPE-1 sequences [14].

Sequence alignment revealed that the different REPs within $X$. campestris and $X$. Oryzae recB genes are located about at the same site in the coding region. In contrast, REPs found in other genes belonging to the same functional category are inserted at different sites. 

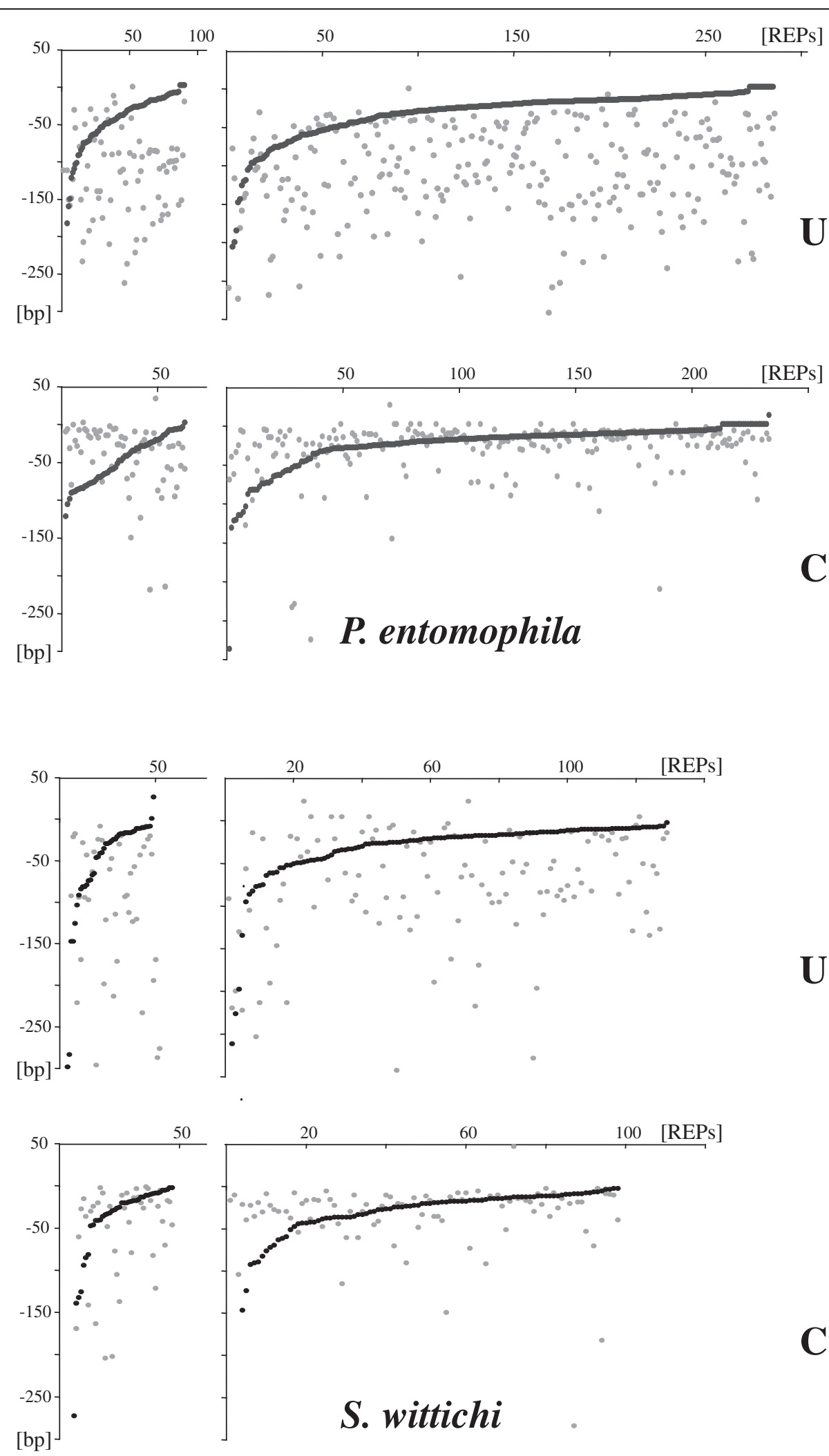

100 [REPs]

Figure 6 (See legend on next page.) 
(See figure on previous page.)

Figure 6 Distances between REPs and flanking ORFs. Dots denote the relative distances from flanking ORFs of uni- and conv-REPs of the $P$. entomophila GTAG-1 and S. wittichi CGTC-1 families. In the uni-REP graphs, upstream and downstream located ORFs are marked as black and gray, respectively. In the conv-REP graphs, the two upstream ORFs are arbitrarily distinguished by the two color code. Single elements and dimers have been separately analyzed. Distances have been sorted by length to facilitate data visualization.

\section{REPs and tyrosine transposases}

GTAG repeats are often found close to genes encoding tyrosine transposases denominated RAYTs [25]. The genetic elements resulting from the association of RAYT and REP sequences are known as REPtrons [51]. REPtrons have been identified in most of the species hosting GTAG repeats listed in Figure 1, as well as in species lacking GTAG repeats (Additional file 4). REPtrons may be missing in some species, because eliminated by deletion as described for many E. coli strains [51].

Species that have multiple GTAG repeats families feature also repeat-specific REPtrons. It is of interest noting that species hosting only one REP family often feature multiple REPtrons. In these, transposase coding sequences, organization and relative position of flanking REPs all vary (Figure 8A; see also Additional file 4).

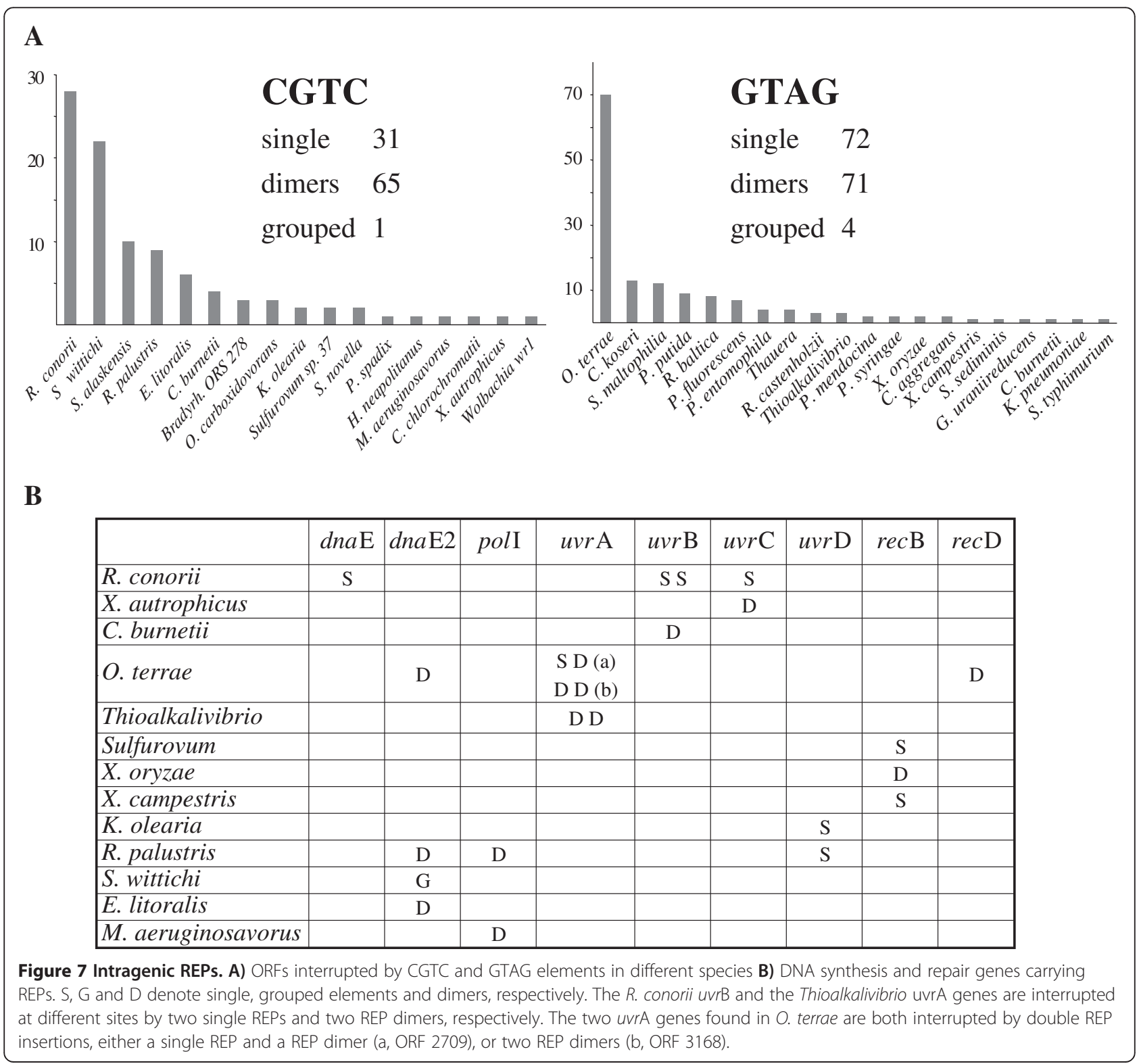


Curiously, REPs are replaced in some REPtrons by long TIRs. TIRs flanking P. putida ppf 607 and P. fluorescens pfs 4255 ORFs result from the adjoining of degenerated GTAG-1 units to unrelated SLSs (Figure 8B), and hundreds of these bizarre structures were found in P. putida and $P$. fluorescens genomes. In contrast, all other TIRs shown in Figure 8A are unrelated to REPs. RAYT genes identified in species that lack GTAG REPs are similarly flanked by TIRs (Figure 9). All these genetic elements and the encoded transposases have been called in accordance TIRtrons and TIRYT (TIR associated tyrosine transposase), respectively. Some TIRs are located about at the same distance from transposase coding sequences, and are plausibly variants of one or a few sequence types, as they share a motif fitting the consensus GGGGWSAS (Figure 9). Other TIRs are unrelated to each other, and some include partly or wholly self-complementary tracts. Moderately abundant families of TIRs have been identified in some microorganisms. Many TIR elements are organized as REPs in dimers or clusters (Figure 9). The highest number of TIR repeats was found in the S. maltophilia K279a strain, which hosts two TIR families, corresponding to the two TIRYT genes ORFs 1152 and 4509. The 1152 and 4509 TIR repeats markedly differ because the former are self-complementary, and are predominantly found at short distance from each other. TIR families of comparable size and organization were found in the other wholly sequenced S. maltophilia strains R551-3, JV3 and D457. Koribacter versatilis has three TIRYT genes (ORFs 1552, 2776, 3477) decorated by different TIRs. Only ORF1552 TIRs are members of a repeated DNA family.

Some of the identified RAYTs, and all the TIRYTs listed in Figure 9, have been aligned for comparison (Additional file 5). The catalytic tyrosine and the HUH (hystidine-hydrophobic-hystidine) domain, typical of transposases of the IS200/IS605 group, are conserved in all, as well as motifs distinguishing RAYTs from bulk IS 200 transposases [25] and other amino acids at several positions. RAYTs and TIRYTs are distinguishable for length and amino acid signatures, and TIRYTs can in turn 


\begin{tabular}{|c|c|c|c|c|c|c|c|c|c|c|}
\hline & & & & & & & & $\mathbf{S}$ & D & G \\
\hline Colwellia psychrerythraea & $\rightarrow 61$ & cps 4446 & 6 & 4 & ATCAAA & GGGGTCAG & AGTtAgTTGATT & - & - & - \\
\hline Methylobacillus flagellatus & $\rightarrow \quad 41$ & $\mathrm{mfa} 477$ & 1 & 4 & AATA & GGGGACAG & ACCACG & - & - & - \\
\hline Nitrosococcus halophilus & $\rightarrow \quad 45$ & nhl 1275 & 45 & 4 & AATATTE & GGGGTCAG & AGTAACAT & - & - & - \\
\hline Shewanella woodyi & $\rightarrow 53$ & \begin{tabular}{|l|} 
swd 1484 \\
\end{tabular} & 21 & 4 & AAA & GGGGTCAG & AGCCCTTTAG & - & - & - \\
\hline Accumulibacter phosphatis & $\rightarrow \quad 23$ & app 1050 & 3 ov & 4 & AAA & GGGGCCAG & GCTCA & - & - & - \\
\hline Thioalkalivibrio K90mix & $\rightarrow \quad 47$ & tkm 1558 & $3^{\prime}$ ov & 4 & GGAAAAC & GGGGACAG & АТтTAтTтTC & - & - & - \\
\hline Mesorhizobium loti & $\rightarrow \quad 42$ & mlo 6832 & $3^{\prime}$ ov & 4 & GAATTAC & GGTGACAG & TGCACT & - & - & - \\
\hline Koribacter versatilis & $\rightarrow 79$ & aba 2776 & 6 & 4 & GGGGAAAATC & GGGGACAG & ACGAAATAAT & - & - & - \\
\hline Hahella chejuensis & $\rightarrow 56$ & hch 1378 & $3^{\prime}$ ov & 4 & $\mathrm{AT}$ & AGGGTCAG & AGTCATTGAT & 5 & - & - \\
\hline Shewanella sediminis & $\rightarrow 56$ & sse 3670 & 16 & 4 & TAAAT & GGGGTCAG & AGTAA & 6 & - & - \\
\hline Marinomonas posidonica & $\rightarrow 67$ & mpc 880 & 21 & $\nabla$ & $\mathrm{T}$ & GGGGTCAG & AGTAAAATTAAA & 6 & - & - \\
\hline Caulobacter crescentus & $\rightarrow 51$ & ccr 1445 & 2 & & $\mathrm{AC}$ & GGtGACAG & TGCACT & 8 & - & - \\
\hline Geobacter sulfurreducens & $\rightarrow \quad 44$ & gsu 2396 & $3^{\prime}$ ov & 4 & AATTCt & GGGGACAC & САТАСТT & - & 1 & - \\
\hline Pseudomonas fluorescens & $\rightarrow \quad 129$ & pfs 148a & 15 & 4 & AAATA & GGGGTCAG & ACCAC & - & 3 & - \\
\hline Bradyrhizobium BTAil & $\rightarrow \quad 40$ & bbt 5382 & 38 & 4 & TTAC & GGtGACAG & TGCA & - & 4 & - \\
\hline Stenotrophomonas maltophilia & $\rightarrow 96$ & sml 4509 & 3 & 4 & AAAA & GGGGACGG & AGGGGATTAAGTCG & 13 & 33 & - \\
\hline Pelobacter carbinolicus & $\rightarrow \quad 52$ & pca 3049 & 3 ov & 4 & & AGGGACAC & TCCCC & 8 & 4 & - \\
\hline Pseudoxanthomonas suwonensis & $\rightarrow 93$ & psu 2716 & 3 ov & & AAAACC & GGGGTCAG & AGTGCAATTTC & 5 & 9 & 66 \\
\hline Desulforudis audaxviator & $\rightarrow \quad 5^{\prime} \mathrm{ov}$ & dau 2153 & 98 & 4 & GGGGTTTCAT & TGT & & - & - & - \\
\hline Granulicella mallensis & $\rightarrow 45$ & gma 794 & 169 & 4 & GCGCGGCAGGC & GTGGGGTATC & & 7 & 5 & - \\
\hline Geobacter bemidjiensis & $\rightarrow 448$ & $\mathrm{gbm} 2474$ & 16 & $\rightarrow 4$ & $\underline{\text { AAAAGGGGACE }}$ & AGGCTACTTT & TTTCTTTTGAAAAAGTAGCCTGTCCCCCTTT & 29 & 22 & - \\
\hline Stenotrophomonas maltophilia & $\rightarrow \leftarrow 123$ & sml 1152 & $3^{\prime}$ ov & $\rightarrow 4$ & GGGGG-CAGA & TCССTTTCGC & CAACGCGGAAGGGATCTGaCCCC & 37 & 79 & 36 \\
\hline Rhodopirellula baltica & $\rightarrow \leftarrow 15$ & rba 2408 & $3^{\prime}$ ov & & AAAAGGTGTCE & :AGGTACCTTT & & 9 & 6 & - \\
\hline Koribacter versatilis & $\rightarrow 42$ & aba 1552 & $3^{\prime}$ ov & & $\overline{\operatorname{ttcGGCAGCGC}}$ & CTGAAGCGCT & TGCCctgatacaga & 30 & 5 & 20 \\
\hline Koribacter versatilis & $\rightarrow<109$ & aba 3477 & 59 & $>4$ & $\underline{\text { TGCCCCAtt } \mathrm{C}^{\prime}}$ & TAAGCTTTCG & GCTC (7-9bp) GAGCGAAAGCTTAGggTGGGGCA & - & - & - \\
\hline Terriglobus saanensis & $\rightarrow 116$ & tsa 392 & 15 & 4 & gggtgc & TGTCCGGATT & TTTCGGACATGGG & 30 & - & - \\
\hline Acidobacterium capsulatum & $\rightarrow 413$ & acp 645 & - & & CCACATCTTC & GCCGAGATGT & TGGG & 22 & - & 8 \\
\hline \multicolumn{11}{|c|}{$\begin{array}{l}\text { Figure } 9 \text { TIRYT genes. TIRs flanking TIRYT genes are diagrammed as arrows. Distances in bp separating genes and TIRs are shown, } 5^{\prime} \text { ov and } 3^{\prime} \text { O } \\
\text { refer to overlapping flanking sequences as in Figure } 8 \text {. Only upstream TIR sequences are shown. Lower case letters denote non complementary } \\
\text { TIR residues. ORFs shown in Figure 8, and conserved GGGGWSAS motifs, are boxed. Complementary residues in the double palindromic TIRs } \\
\text { shown at the bottom are underlined. Boxed numbers to the right refer to single (S), dimeric (D) or grouped (G) TIR sequences found in the } \\
\text { indicated genomes. }\end{array}$} \\
\hline
\end{tabular}

be assigned to four main groups (Additional file 5). Of these, the more sharply defined is represented by the transposases encoded by T. saanensis (tsa 392), K. versatilis (aba 1552 and 3447), A. capsulatum (acp 645) and G. mallensis (gma794), species all belonging to the Acidobacteria phylum.

In spite of the overall similarity to GTAG elements, CGTC repeats are not associated to transposase genes. Many of the CGTC-positive species in Figure 2, among which Bradyrhizobium sp. ORS278, C. crescentus, C. taiwanensis, G. forsetii, R. palustris. Sulfurovum sp. NBC37-1, K. olearia, P. spadix, S. lithotrophicus, encode tyrosine transposases, but none of the corresponding genes were flanked by CGTC sequences. The interspersion of CGTC elements with other classes of transposase genes was also monitored, but only a few fortuitous associations have been detected.

\section{Discussion}

Data reported in this work support the notion that many short palindromic repeats found in prokaryotes may be evolutionarily related, and catalogued as members of two large DNA super-families alternatively tagged at one end by GTAG or CGTC motifs not involved in base pairing. Distinctive features of GTAG and CGTC repeats are summarized in Table 1. GTAG and CGTC superfamilies include more sequence classes than those reported. Members of either type may have escaped detection because: 1) smaller than average repeats. Thauera GTAG-1 elements, which feature only 5 bp stems, were fortuitously discovered by inspection of the tandem repeat database [52] 2) unusual in structure, for the presence of bulges due to unpaired residues 3 ) poorly recognizable, as the degenerated Pseudomonas GTAG-1 repeats shown in Figure 8. The data presented are however sufficient to draw a coherent picture of the

\section{Table 1 Features of REP families}

\begin{tabular}{ccc}
\hline & GTAG & CGTC \\
\hline GW extra-bases & GTAG-1, 2 & - \\
3 bp tail & GTAG-1, 2 & all \\
stem length & $5-13 \mathrm{bp}$ & $8-9 \mathrm{bp}$ \\
loop length & $2-20 \mathrm{bp}$ & $4-5 \mathrm{bp}$ \\
clusters & frequent & rare \\
HH dimers & predominant & predominant \\
TT dimers & frequent & rare \\
intragenic units & + & + \\
association to TPAse genes & + & - \\
\hline
\end{tabular}


organization of GTAG and CGTC repeats, evaluate the pattern of distribution of the various families among species, reexamine the roles that these sequences may play, shed light on the processes by which they might have been formed.

GTAG and CGTC REP families vary in size over a 50-fold range, some including thousands units, many 20-100 units, or even less, and are unevenly distributed among species. Both observations rule out that these elements may be important chromosome components fulfilling the same general functions in all organisms $[8,10]$. In contrast, the beneficial effects on host fitness may vary in different environments, and in some microorganisms specific repeats may just be parasitic DNA. GTAG and CGTC elements come in different chromosomal arrangements. The relative abundance of single, paired and clustered elements within each family varies among species, as among isolates of the same species, and changes in the organization of family units are genomic fingerprints exploitable for genotyping assays [53].

Most of the described REPs are located in the intergenic space. Taking into account that the average intergenic space in prokaryotes is -100 bp [54], many are close to, or overlap with coding regions. The preferential location between unidirectionally and convergently transcribed ORFs, and the frequency of GT pairing of stem residues, both support the notion that many repeats are transcribed, and may function as posttranscriptional control sequences, by tuning the levels of expression of flanking genes.

REPs may as well function as DNA elements. The $E$. coli REPs are targeted by the DNA gyrase [10], and cleavage of REPs located at ORF 3' ends by gyrase may relieve the excess of supercoiling induced by transcription [55]. This regulatory mechanism would however be effective only in REP-rich species. Other repeats may function as promoters in specific microorganisms and/or genomic contexts. The issue has not been tackled, because promoter analyses without experimental support are merely speculative. Yet, it is worth noting that, analyzing the interspersion of GTAG-1 elements with coding regions in the exopolysaccharide (EPS)-producing bacterium Thauera sp. MZ1T, we unexpectedly found that clustered genes involved in EPS synthesis and transport [56] are immediately flanked by arrays of GTAG-1 repeats, which likely direct or modulate their expression.

In different organisms GTAG and CGTC REPs have been found within coding regions, most of which encode known proteins. It is difficult to assess whether intragenic elements may affect the activity of the decorated proteins. The insertion of REPs in a variety of unrelated proteins argues against functional constraints, and genes inactivated by REP insertions have been plausibly removed from the population. Amino acids encoded by intragenic elements found at the $\mathrm{NH} 2$ - or the $\mathrm{COOH}$ terminus may not affect the function of the protein. Moreover, most REPs located in the NH2-terminal coding region may be extragenic, because of genome misannotation. An additional argument against the inactivating role that REP insertions may play is that tagged proteins may have modular structure, and insertions may be neutral in effect, because located in flexible linkers or loops. In spite of all these cautions, it is difficult hypothesize that genes encoding different proteins involved in replication and global genome repair (UvrABCD and recBCD proteins, DNA polymerase I, error prone DNA polymerases) may have been just fortuitously targeted by REP insertions, also because they are, in many species, the only examples of REP-tagged coding sequences. It is therefore tempting to speculate that insertions may have modified the activity of the mentioned proteins, contributing to the development of hypermutable or mutator microorganisms, which may experience increased recombination, mutation, gene loss, horizontal gene transfer. Multiple tRNA pseudouridine synthase genes also carry REP sequences, but is unclear how these insertions may affect cell physiology. Pseudouridine synthases are involved in posttranscriptional modifications of cellular RNA, but act also as RNA chaperones, a function which may be more important than pseudouridylation per se [57].

The occurrence in multiple distant phyla supports the notion that both GTAG and CGTC repeats are ancient components of the bacterial genome. Most elements reside in Proteobacteria, and GTAG and CGTC repeats have been predominantly identified in the gamma and alpha division, respectively. However, families of either repeat type have been identified in deeper branching phyla among which Termotogae and Planctomycetes, plausibly the deepest branching phylum within the bacterial domain [58]. Planctomycetes cluster with Verrucomicrobia in the PVC superphylum, and O. terrae, which belongs to Verrucomicrobia, is highly enriched in GTAG repeats. Bacterial phyla are related to each other linearly, and major evolutionary changes within Bacteria have taken place in a directional manner [28]. REPs plausibly appeared early in evolution, and have been massively lost in time, and maintained in a limited number of microorganisms. How all this occurred is a matter of speculation. Though the actual scenario will likely be modified by analyzing a wider set of genomes, the distribution of REPs described in this work among phyla, orders, families and species is manifestly uneven. GTAG repeats have been identified in microorganisms belonging to 10 of the 15 orders of gamma-Proteobacteria (Figure 1). In turn, only one of a few species within each 
order host GTAG repeats. Enterobacteria have been subdivided into three clusters on the basis of the character states of aromatic amino acid biosynthesis [59]. Cluster 1 includes Escherichia, Shigella, Citrobacter, Salmonella, Klebsiella, Enterobacter, cluster 2 Serratia and Erwinia, cluster 3 Edwardsiella, Yersinia, Proteus and Providencia. GTAG-3 families are sharply confined to species of enterocluster 1. Similarly, GTAG repeats reside only in some species of the genus Shewanella. Shewanellae fall into two major clusters based on their 16S rDNA sequences as well as phenotypic properties [60]. Cluster I includes cold-adapted obligate marine species retrieved from the deep sea, cluster II non-obligate marine species retrieved from different environments. Interestingly, GTAG-1 and GTAG-2 families have been identified only in species (S. sediminis, S. halifaxensis, S. pealeana, S. woodyi and S. piezotolerans) belonging to cluster I. The above reported examples suggest that the presence/absence of specific REP families may represent a resource exploitable to catalogue bacteria, useful to support, or weaken, phylogenetic relatedness among groups of microorganisms inferred by the use of conventional parameters. CGTC repeats are unevenly distributed among species as well. As an example, CGTC repeats have been identified in all orders of the alpha subdivision, but are missing in several alpha-Proteobacteria, among which bacteria belonging to the families of Acetobacteraceae, Bartonellaceae and Brucellaceae.

The abundant families of GTAG repeats are restricted both in S. maltophilia [9] and P. syringae [61] to core genome regions. Yet, the spotty distribution is compatible with the hypothesis that specific genomes may have been colonized by REPs as a consequence of HGT (horizontal gene transfer) events. According to this view, repeats must have been acquired along with genes ensuring their multiplication. Differences in the distribution and abundance of REPs among different species, or strains of the same species, are typical of mobile DNA. Different groups in the recent past suggested that REPs are selfish elements propagated by transposition. A key role in the process is (or has been) played by specific tyrosine transposases called RAYTs. Transposon-like elements including REP and RAYT sequences called REPtrons have been identified in a variety of species, regardless the presence of a corresponding REP family. Whether the expression of RAYTs in these elements is driven by REPs is unknown, but marked differences in the organization of REPtrons, as the inability of REPtrons to self-propagate, do not support such hypothesis. The expression of RAYTs is plausibly correlated to the formation of upstream readthrough transcripts, and can be indeed down-regulated by hairpins formed by REPs, which may either promote mRNA degradation, or affect mRNA translation, as observed for IS200 transposases [62]. Direct involvement of RAYTs in the formation of REPs is supported by experiments showing that a recombinant $E$. coli RAYT recognizes single-stranded REP DNA, and cleaves the GTAG motif [51,63]. Cleavage was abolished by mutating the motif, or changing the AA/GC residues at the edges of the loop region (see Figure 1) into paired AA/TT residues, thus by increasing the strength of the REP palindrome. In the model proposed [51] REP sequences are the products of RAYT-mediated excision and recombination events, and $\mathrm{HH}$ or TT dimers, or complex REP arrays may result from alternative processing of circular intermediates carrying REP units. GTAG-1 and GTAG-2 repeats carry conserved 3-bp sequences at the untagged end. Whether these "tails" are recognized by RAYTs, and similar signals are present but have been variously altered in other repeat families remains to be established.

Comparative analyses revealed that several RAYT-like genes are not flanked by REPs, but rather by TIRs of different length and composition. These transposases and the corresponding genetic structures have been called for consistency TIRYTs and TIRtrons, respectively. TIRtrons occur in species which contain REPs, but are predominant in species which lack REPs. Given the extraordinary high number of annotated tyrosine transposase genes (at the moment, $>2000$ ), it is likely that many REPtron- and TIRtron-like entities occur. Unravelling the complexity of this variegated universe of sequences is out of the scope of this work. Yet, monitoring TIRtrons and similar entities may shed light on the process of formation of REPs, since TIRs flanking some TIRYT genes are members of previously undiscovered repeated DNA families. The formation of TIR and GTAG REP families could thus be mediated by TIRYTs and RAYTs, and occur in an analogous manner. In contrast to REPtrons and REPs, TIRtrons and TIRs coexist in a limited number of genomes, suggesting that TIRYTs may be less productive players than RAYTs.

There is no obvious correlation between the presence of tyrosine transposase genes and the occurrence of REP or TIR families. $K$. versatilis has three distinct TIRYT genes (ORFs aba 2776, 3477, and 1552; see Figure 9), and one family of TIR repeats, A. phosphatis two different TIRYTs, ORFs app 1050 (Figure 9) and app 3234 (not shown), but no TIR repeats. In contrast, a plethora of tyrosine tranposase genes and corresponding flanking repeats was found in $P$. fluorescens, $R$. baltica and $S$. maltophilia. This suggests that the formation and/or maintenance of repeats promoted by tyrosine tranposase may be favored in specific microorganisms.

Functional interactions of recombinant RAYTs and TIRYTs with REP and TIR targets may be eventually analyzed to check whether RAYTs can bind and/or cleave TIR repeats, and vice versa, whether TIRYTs recognize GTAG repeats. The variety of REP and TIR targets, and 
the occurrence of a multitude of element-specific transposases, make $S$. maltophilia a reference organism to set up in vitro assays. For the same reasons, it should be of interest to assess the mobility of GTAG and TIR repeats by population sequencing, as elegantly done to monitor transposition of GTAG-1 repeats in Pseudomonas [26].

CGTC elements markedly differ from GTAG repeats because seem lacking a dedicated transposase. Genes encoding RAYT and other IS200 transposases reside in many of the species carrying CGTC repeats, but none of them is flanked by CGTC units. Such marked difference between GTAG and CGTC elements could be explained by hypothesizing that CGTC REPtrons may have early disappeared, plausibly because able to propagate very efficiently, and therefore highly deleterious to the host. According to this view, the formation of novel repeats is blocked, and CGTC families are going toward extinction. Alternatively, the absence of a dedicated enzyme may imply that CGTC elements can be mobilized by a broad spectrum of transposases. The two hypotheses are not in contrast, and CGTC-specific transposases may have been replaced by functionally related enzymes.

\section{Conclusions}

The provisional framework provided by this paper sets the base for a coherent classification scheme according to which catalogue several small palindromic repeats found in prokaryotes. Future work should clarify the degree of relatedness of CGTC and GTAG repeats, assess whether they have been formed by similar processes, and if such processes are still operative. The relatedness of tagged and untagged SLSs also needs to be investigated. Families of REP-like sequences lacking conserved terminal motifs have been identified in $M$. tuberculosis and $D$. radiodurans [8], Bordetellae [64], Brucellae [44] and Cyanobacteria [65], but many more likely occur. It will be of interest to assess whether classes of untagged palindromic repeats may be evolutionarily related, and functionally associated with specific DNA- or RNA-binding proteins.

\section{Methods}

\section{DNA analyses}

DNA sequences analyzed in this work include known and novel repeats. The names and the NCBI accession numbers of all the genomes analyzed in this study are listed in Additional file 6. Novel repeats have been identified by BLAST, using as queries known REPs variously modified, or sets of 20 mers featuring 7-8 base paired residues, separated by loops of variable lengths. Some repeats were identified by searching abundant, self-complementary sequences in individual prokaryotic genomes by using the TRDB (Tandem Repeats Database) facility [52].
The organization of the various repeat families was assessed by using the Fuzznuc program of the EMBOSS package. Genomes of interest were searched for SLSs homologous to queries known or derived from BLAST searches, containing mismatches and a variable number of loop residues. In the pruning procedure, palindromic repeats containing more than one mismatch in the paired region were discarded, but retained when repeats were partners of dimers. GT pairing between stem residues was allowed. Repeats with loops unusual for length or composition relatively to the majority of family members were also discarded. The extent of variation of REP families among different species, or isolates of the same species, was determined by comparing the relative abundance of the major sequence types or subsets identified in representative genomes.

\section{Additional files}

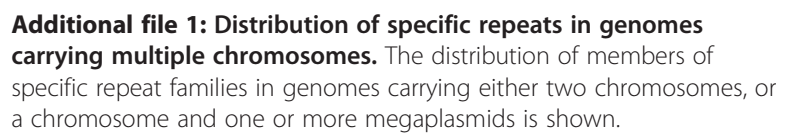

Additional file 2: Distance between REPs and flanking ORFs in REP-rich species. Distances separating REPs from flanking ORFs in four REP-rich species (P. putida, C. koseri, S. novella and S. alaskensis) are shown. Data are presented as in Figure 6.

Additional file 3: Intragenic REPs. The number, the size in amino acids and the hypothesized function of ORFs carrying GTAG and CGTC elements are shown. For each, the interval encoded by REP sequences and the corresponding amino acids are shown.

Additional file 4: REPtrons list. Tyrosine transposase genes not included in Figure 8 are shown. The sequences of REP-like elements decorating REPtrons found in species lacking REP families are also shown.

Additional file 5: Alignment of RAYT and TIRYTs. Some of the identified RAYTs, and all the TIRYTs listed in Figure 9, have been aligned for comparison.

Additional file 6: Full name and NC accession number of the analyzed strains.

\begin{abstract}
Abbreviations
bp: Base pair; BIME: Bacterial interspersed mosaic element; BLAST: Basic local alignment sequence tool; CRISPR: Clustered regularly interspaced short palindromic repeat; EPS: Exopolysaccharide; HGT: Horizontal gene transfer; HUH: Hystidine-hydrophobic-hystidine; IS: Insertion sequence; Kb: Kilo base; MITE: Miniature inverted-repeat transposable element; ORF: Open reading frame; PVC: Planctomycetes, verrucomicrobia and chlamydiales; RAYT: REPassociated tyrosine transposase; REP: Repetitive extragenic palindrome; RPE: Repetitive palindromic element; SLS: Stem-loop sequence; ST: Sequence type; TIR: Terminal inverted repeat; TIRYT: TIR-associated tyrosine transposase; TRDB: Tandem repeats database.
\end{abstract}

Competing interests

The authors declare that they have no competing interests.

\section{Authors' contributions}

PPDN conceived the study and wrote the manuscript, FR analyzed the composition of REP families, EDG analyzed intragenic elements and dimer repeats, and prepared all graphic work. All authors read and approved the manuscript. 


\section{Acknowledgments}

We are indebted to Prof. Raffaele Zarrilli for suggestion and critical reading of the manuscript. This research was supported by a grant assigned to Pier Paolo Di Nocera by the PRIN 2009 agency of the Italian Ministry of University and Scientifc Research.

Received: 6 May 2013 Accepted: 30 July 2013

Published: 31 July 2013

\section{References}

1. Siguier P, Filée J, Chandler M: Insertion sequences in prokaryotic genomes. Curr Opin Microbiol 2006, 9:526-531.

2. Touchon M, Rocha EP: Causes of insertion sequences abundance in prokaryotic genomes. Mol Biol Evol 2007, 24:969-981.

3. Delihas N: Impact of small repeat sequences on bacterial genome evolution. Genome Biol Evol 2011, 3:959-973.

4. Marraffini LA, Sontheimer EJ: CRISPR interference: RNA-directed adaptive immunity in bacteria and archaea. Nat Rev Genet 2010, 11:181-190.

5. Bachellier S, Clement JM, Hofnung M: Short palindromic repetitive DNA elements in enterobacteria: a survey. Res Microbiol 1999, 150:627-639.

6. Aranda-Olmedo I, Tobes R, Manzanera M, Ramos JL, Marques S: Species-specific repetitive extragenic palindromic (REP) sequences in Pseudomonas putida. Nucleic Acids Res 2002, 30:1826-1833.

7. Feil H, Feil WS, Chain P, Larimer F, DiBartolo G, Copeland A, Lykidis A, Trong S, Nolan M, Goltsman E, et al: Comparison of the complete genome sequences of Pseudomonas syringae pv. syringae B728a and pv. tomato DC3000. Proc Natl Acad Sci U S A 2005, 102:11064-11069.

8. Tobes R, Ramos JL: REP code: defining bacterial identity in extragenic space. Environ Microbiol 2005, 7:225-228.

9. Rocco F, De Gregorio E, Di Nocera PP: A giant family of short palindromic sequences in Stenotrophomonas maltophilia. FEMS Microbiol Lett 2010, 308:185-192.

10. Higgins CF, McLaren RS, Newbury SF: Repetitive extragenic palindromic sequences, mRNA stability and gene expression: evolution by gene conversion? A review. Gene 1988, 72:3-14.

11. Espéli $\mathrm{O}$, Moulin L, Boccard F: Transcription attenuation associated with bacterial repetitive extragenic BIME elements. J Mol Biol 2001, 314:375-386.

12. Tobes R, Pareja E: Bacterial repetitive extragenic palindromic sequences are DNA targets for insertion sequence elements. BMC Genomics 2006, 7:62.

13. Ogata H, Audic S, Abergel C, Fournier PE, Claverie JM: Protein coding palindromes are a unique but recurrent feature in Rickettsia. Genome Res 2002, 12:808-816.

14. Claverie JM, Ogata $\mathrm{H}$ : The insertion of palindromic repeats in the evolution of proteins. Trends Biochem Sci 2003, 28:75-80

15. Oggioni M, Claverys JP: Repeated extragenic sequences in prokaryotic genomes: a proposal for the origin and dynamics of the RUP element in Streptococcus pneumoniae. Microbiology 1999, 145:2647-2653.

16. Mazzone M, De Gregorio E, Lavitola A, Pagliarulo C, Alifano P, Di Nocera PP: Whole-genome organization and functional properties of miniature DNA insertion sequences conserved in pathogenic Neisseriae. Gene 2001, 278:211-222.

17. De Gregorio E, Abrescia C, Carlomagno MS, Di Nocera PP: Ribonuclease III-mediated processing of specific Neisseria meningitidis mRNAs. Biochem J 2003, 374:799-805.

18. Okstad OA, Tourasse NJ, Stabell FB, Sundfaer CK, Egge-Jacobsen W, Risoen PA, Read TD, Kolsto AB: The bcr1 DNA repeat element is specific to the Bacillus cereus group and exhibits mobile element characteristics. J Bacteriol 2004, 186:7714-7725.

19. De Gregorio E, Silvestro G, Petrillo M, Carlomagno MS, Di Nocera PP: Enterobacterial repetitive intergenic consensus sequence repeats in Yersiniae: genomic organization and functional properties. J Bacteriol 2005, 187:7945-7954.

20. De Gregorio E, Silvestro G, Venditti R, Carlomagno MS, Di Nocera PP: Structural organization and functional properties of miniature DNA insertion sequences in Yersiniae. J Bacteriol 2006, 188:7876-7884.

21. Zhou F, Tran T, Xu Y: Nezha, a novel active miniature inverted-repeat transposable element in cyanobacteria. Biochem Biophys Res Commun 2008, 365:790-794.
22. De Gregorio E, Bertocco T, Silvestro G, Carlomagno MS, Zarrilli R, Di Nocera PP: Structural organization of a complex family of palindromic repeats in Enterococci. FEMS Microbiol Lett 2009, 292:7-12.

23. Delihas N: Small mobile sequences in bacteria display diverse structure/ function motifs. Mol Microbiol 2008, 67:475-481.

24. Bardaji L, Añorga M, Jackson RW, Martínez-Bilbao A, Yanguas-Casás N, Murillo J: Miniature transposable sequences are frequently mobilized in the bacterial plant pathogen Pseudomonas syringae pv. phaseolicola. PLoS One 2011, 6:e25773.

25. Nunvar J, Huckova T, Licha I: Identification and characterization of repetitive extragenic palindromes (REP)-associated tyrosine transposases: implications for REP evolution and dynamics in bacterial genomes. BMC Genomics 2010, 11:44.

26. Bertels F, Rainey PB: Within-genome evolution of REPINs: a new family of miniature mobile DNA in bacteria. PLoS Genet 2011, 7:e1002132.

27. Wagner M, Horn M: The planctomycetes, verrucomicrobia, chlamydiae and sister phyla comprise a superphylum with biotechnological and medical relevance. Curr Opin Biotechnol 2006, 17:241-249.

28. Gupta RS: The natural evolutionary relationships among prokaryotes. Crit Rev Microbiol 2000, 26:111-131.

29. Schirrmeister BE, Antonelli A, Bagheri HC: The origin of multicellularity in bacteria. BMC Evol Biol 2011, 14:11-45.

30. Omsland A, Heinzen RA: Life on the outside: the rescue of Coxiella burnetii from its host cell. Annu Rev Microbiol 2011, 65:111-128.

31. Ge Q, Ilves H, Dallas A, Kumar P, Shorenstein J, Kazakov SA, Johnston BH: Minimal-length short hairpin RNAs: the relationship of structure and RNAi activity. RNA 2010, 16:106-117.

32. Gupta RS, Mok A: Phylogenomics and signature proteins for the alpha Proteobacteria and its main groups. BMC Microbiol 2007, 7:106.

33. Wang Z, Kadouri DE, Wu M: Genomic insights into an obligate epibiotic bacterial predator: Micavibrio aeruginosavorus ARL-13. BMC Genomics 2011, 12:453.

34. Schoen C, Joseph B, Claus H, Vogel U, Frosch M: Living in a changing environment: insights into host adaptation in Neisseria meningitidis from comparative genomics. Int J Med Microbiol 2007, 297:601-613.

35. Ogata $\mathrm{H}$, Suhre $\mathrm{K}$, Claverie JM: Discovery of protein-coding palindromic repeats in Wolbachia. Trends Microbiol 2005, 13:253-255.

36. Parkhill J, Achtman M, James KD, Bentley SD, Churcher C, Klee SR, Morelli G, Basham D, Brown D, Chillingworth T, et al: Complete DNA sequence of a serogroup A strain of Neisseria meningitidis Z2491. Nature 2000 404:502-506.

37. Lindeberg M, Cartinhour S, Myers CR, Schechter LM, Schneider DJ, Collmer A: Closing the circle on the discovery of genes encoding Hrp regulon members and type III secretion system effectors in the genomes of three model Pseudomonas syringae strains. Mol Plant Microbe Interact 2006, 19:1151-1158.

38. Silby MW, Cerdeño-Tárraga AM, Vernikos GS, Giddens SR, Jackson RW, Preston GM, Zhang XX, Moon CD, Gehrig SM, Godfrey SA, et al: Genomic and genetic analyses of diversity and plant interactions of Pseudomonas fluorescens. Genome Biol 2009, 10:R51.

39. Wu X, Monchy S, Taghavi S, Zhu W, Ramos J, van der Lelie D: Comparative genomics and functional analysis of niche-specific adaptation in Pseudomonas putida. FEMS Microbiol Rev 2011, 35:299-323.

40. Giraud E, Moulin L, Vallenet D, Barbe V, Cytryn E, Avarre JC, Jaubert M, Simon D, Cartieaux F, Prin Y, et al: Legumes symbioses: absence of Nod genes in photosynthetic bradyrhizobia. Science 2007, 316:1307-1312.

41. Simmons SS, Isokpehi RD, Brown SD, McAllister DL, Hall CC, McDuffy WM, Medley TL, Udensi UK, Rajnarayanan RV, Ayensu WK, Cohly HH: Functional annotation analytics of rhodopseudomonas palustris genomes. Bioinform Biol Insights 2011, 5:115-129.

42. Bandyopadhyay A, Elvitigala T, Welsh E, Stöckel J, Liberton M, Min H, Sherman LA, Pakrasi HB: Novel metabolic attributes of the genus cyanothece, comprising a group of unicellular nitrogen-fixing Cyanothece. Mbio 2011, 2:e00214-11.

43. McLeod MP, Qin X, Karpathy SE, Gioia J, Highlander SK, Fox GE, McNeill TZ, Jiang H, Muzny D, Jacob LS, et al: Complete genome sequence of Rickettsia typhi and comparison with sequences of other rickettsiae. J Bacteriol 2004, 186:5842-5855.

44. Cozzuto L, Petrillo M, Silvestro G, Di Nocera PP, Paolella G: Systematic identification of stem-loop containing sequence families in bacterial genomes. BMC Genomics 2008, 9:20 
45. Bzymek M, Lovett ST: Instability of repetitive DNA sequences: the role of replication in multiple mechanisms. Proc Natl Acad Sci USA 2001, 98:8319-8325.

46. Sinden RR, Zheng GX, Brankamp RG, Allen KN: On the deletion of inverted repeated DNA in Escherichia coli: effects of length, thermal stability, and cruciform formation in vivo. Genetics 1991, 129:991-1005.

47. Connelly JC, de Leau ES, Leach DR: DNA cleavage and degradation by the SbcCD protein complex from Escherichia coli. Nucleic Acids Res 1999, 27:1039-1046.

48. Boccard F, Prentki P: Specific interaction of IHF with RIBs, a class of bacterial repetitive DNA elements located at the $3^{\prime}$ end of transcription units. EMBO J 1993, 12:5019-5027.

49. Abergel C, Blanc G, Monchois V, Renesto P, Sigoillot C, Ogata H, Raoult D, Claverie JM: Impact of the excision of an ancient repeat insertion on Rickettsia conorii guanylate kinase activity. Mol Biol Evol 2006, 23:2112-2122.

50. Erill I, Campoy S, Mazon G, Barbé J: Dispersal and regulation of an adaptive mutagenesis cassette in the bacteria domain. Nucleic Acids Res 2006, 34:66-77.

51. Ton-Hoang B, Siguier P, Quentin Y, Onillon S, Marty B, Fichant G, Chandler $M$ : Structuring the bacterial genome: $Y 1$-transposases associated with REP-BIME sequences. Nucleic Acids Res 2012, 40:3596-3609.

52. Gelfand Y, Rodriguez A, Gary Benson G: TRDB-The Tandem Repeats Database. Nucleic Acids Res 2007, 35:D80-D87.

53. Roscetto E, Rocco F, Carlomagno MS, Casalino M, Colonna B, Zarrilli R, Di Nocera PP: PCR-based rapid genotyping of Stenotrophomonas maltophilia isolates. BMC Microbiol 2008, 8:202.

54. Koonin EV, Wolf Yl: Genomics of bacteria and archaea: the emerging dynamic view of the prokaryotic world. Nucleic Acids Res 2008, 36:6688-6719.

55. Moulin L, Rahmouni AR, Boccard F: Topological insulators inhibit diffusion of transcription-induced positive supercoils in the chromosome of Escherichia coli. Mol Microbiol 2005, 55:601-610.

56. Jiang K: Genomic and Molecular Analysis of the Exopolysaccharide Production in the Bacterium Thauera aminoaromatica MZ1T. PhD thesis. University of Tennessee; 2011

57. Hamma T, Ferré-D'Amaré AR: Pseudouridine synthases. Chem Biol 2006, 13:1125-1135

58. Brochier C, Philippe H: Phylogeny: a non-hyperthermophilic ancestor for bacteria. Nature 2002, 417:244.

59. Ahmad S, Weisburg WG, Jensen RA: Evolution of aromatic amino acid biosynthesis and application to the fine-tuned phylogenetic positioning of enteric bacteria. J Bacteriol 1990, 172:1051-1061.

60. Zhao JS, Deng Y, Manno D, Hawari J: Shewanella spp. genomic evolution for a cold marine lifestyle and in-situ explosive biodegradation. PLoS One 2010, 5:e9109.

61. Tobes R, Pareja E: Repetitive extragenic palindromic sequences in the Pseudomonas syringae pv. tomato DC3000 genome: extragenic signals for genome reannotation. Res Microbiol 2005, 156:424-433.

62. Beuzón CR, Chessa D, Casadesús J: IS200: an old and still bacterial transposon. Int Microbiol 2004, 7:3-12.

63. Messing SA, Ton-Hoang B, Hickman AB, McCubbin AJ, Peaslee GF, Ghirlando R, Chandler M, Dyda F: The processing of repetitive extragenic palindromes: the structure of a repetitive extragenic palindrome bound to its associated nuclease. Nucleic Acids Res 2012, 40:9964-9979.

64. Petrillo M, Silvestro G, Di Nocera PP, Boccia A, Paolella G: Stem-loop structures in prokaryotic genomes. BMC Genomics 2006, 7:170.

65. Elhai J, Kato M, Cousins S, Lindblad P, Costa JL: Very small mobile repeated elements in cyanobacterial genomes. Genome Res 2008, 18:1484-1499.

doi:10.1186/1471-2164-14-522

Cite this article as: Di Nocera et al:: GTAG- and CGTC-tagged palindromic DNA repeats in prokaryotes. BMC Genomics 2013 14:522.

\section{Submit your next manuscript to BioMed Central and take full advantage of:}

- Convenient online submission

- Thorough peer review

- No space constraints or color figure charges

- Immediate publication on acceptance

- Inclusion in PubMed, CAS, Scopus and Google Scholar

- Research which is freely available for redistribution 